\title{
The signature of inner-core nucleation on the geodynamo
}

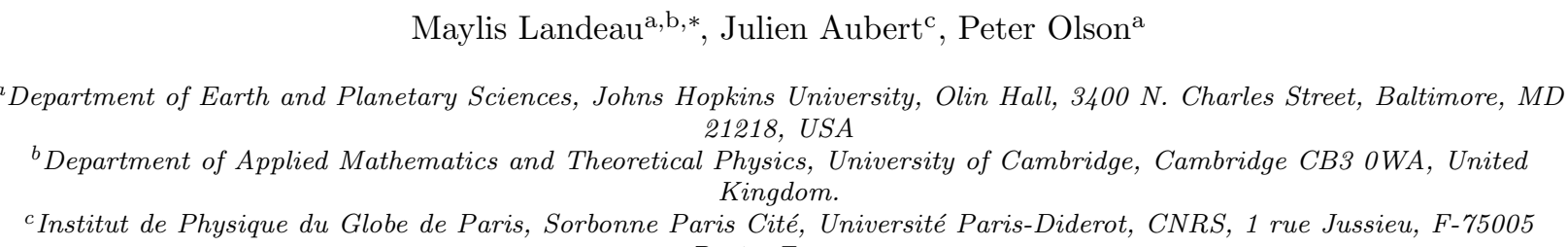

\section{Abstract}

Energy considerations indicate that the power delivered to the present-day geodynamo comes mainly from the growth of the solid inner core, through light element and latent heat releases. The nucleation of the inner core was, therefore, a major transition for the geodynamo. Here, we use numerical dynamo simulations linked by thermochemical evolution of the core to investigate the effects of inner-core nucleation (ICN) on the geodynamo, and identify possible ICN footprints in the palaeomagnetic field. Our results suggest that a strong-field dipole-dominated dynamo prior and after ICN (the uniformitarian scenario) is most likely. We predict little footprint of ICN on the surface field intensity, consistent with the observed lack of a long-term trend in paleointensity data. Instead, we find that the best proxy for inner-core growth is an increase in axial octupole strength with age, from present-day to ICN, which might be resolvable in the palaeomagnetic record. We confirm the existence of weak-field, multipolar dynamos prior to ICN, suggesting the dynamo might have transiently reached a weak-field state (the catastrophic scenario). The narrow stability domain of these weak-field dynamos implies that the catastrophic scenario is less probable than the uniformitarian scenario.

Keywords: geodynamo; weak-field vs strong-field dynamo; inner-core nucleation; geomagnetic field on long time scales; paleointensity; Geocentric Axial Dipole.

\section{Introduction}

The time at which Earth's inner core nucleated marked a major transition for core history, not only affecting the structure of the core but also inducing a sharp change in core energetics and in the powering of the geodynamo. Energy considerations indicate that the present-day geodynamo is mainly powered by inner-core solidification, through latent heat and light element releases at the inner-core boundary (ICB) (Lister, 2003; Labrosse, 2015). In contrast, before the nucleation of a solid inner core, the geodynamo was powered by secular cooling alone, possibly helped by magnesium precipitation (O'Rourke and Stevenson,

*Corresponding author; email: landeau@damtp.cam.ac.uk Preprint submitted to Elsevier

December 25, 2016 
2016; Badro et al., 2016) or tidal deformations (Le Bars et al., 2011). As a result, the power available for the dynamo was smaller prior to ICN than at present-day by a factor up to 100 (Stevenson et al., 1983; Labrosse, $2003)$.

Core thermal evolution models, which are based on energy and entropy balances in the core (e.g. Labrosse, 2003; Lister, 2003), provide constraints on the age of ICN. However, the resulting range of possible values is broad, especially with the ongoing controversies about the value of core thermal conductivity (de Koker et al., 2012; Pozzo et al., 2013; Gomi et al., 2013; Ohta et al., 2016; Konôpková et al., 2016). Recent studies, based on first-principle computations (Gomi et al., 2013; Ohta et al., 2016) or experiments (Gomi et al., 2013; Ohta et al., 2016), point towards a high value of the thermal conductivity two or three times higher than previous estimates; however, experiments by Konôpková et al. (2016) suggest a much lower thermal conductivity. Most recent core evolution models that use upward revision of the thermal conductivity predict a young inner core, with ICN age smaller than 1-1.5 Ga (Labrosse, 2015; Davies, 2015; Olson et al., 2015).

Meanwhile, advances in paleointensity measurements on single grain samples have revealed that the geodynamo was active 3.5 Ga (Tarduno et al., 2010; Biggin et al., 2011) or even 4.2 Ga ago (Tarduno et al., 2015), indicating that ICN likely occured during a time covered by palaeomagnetic data.

The above developments pose multiple, still unresolved, questions about the history of the geodynamo. The question that has received the most attention is whether ICN induced changes in magnetic field properties, leaving footprints in the palaeomagnetic record, and providing independent constraints on ICN age. Another question, perhaps more fundamental than the first, is how to explain the enigmatic lack of uncontroversial trend in the paleointensity record during the last two billion years (Smirnov et al., 2016).

In this paper, we use a series of numerical dynamo simulations linked by core energetics, in order to investigate the effects of ICN on the geodynamo and address the above questions. In the following, we assume that thermal convection was the only power source for the geodynamo during the several hundred million years that preceeded ICN, a plausible scenario according to thermal evolution models (Driscoll and Bercovici, 2014; Olson et al., 2015).

\section{State of the art on ICN palaeomagnetic signatures}

It has long been proposed that the sharp change in power at ICN could have left a footprint in the paleointensity record, with a weaker field prior to ICN and a stronger field just after ICN (Stevenson et al., 1983; Hale, 1987). This expected drop in field intensity with age motivated hunts for ICN in the paleointensity record (e.g. Hale, 1987; Valet et al., 2014; Biggin et al., 2015). A few investigations have claimed to detect a decrease in field intensity with age, interpreted as a footprint of ICN, but its location varies from one study to the other: Biggin et al. (2015) locate the decrease between $1 \mathrm{Ga}$ and $1.5 \mathrm{Ga}$, with a field minimum around 1.5 
Ga, Macouin et al. (2004) argue for a decrease within 0.3 - $1 \mathrm{Ga}$, and Valet et al. (2014) find a field minimum around $1.8-2.3 \mathrm{Ga}$. Other palaeomagnetic investigations argue for the absence of clear long-term trend in the paleointensity record within the Precambrian period (Selkin and Tauxe, 2000; Tauxe, 2006; Smirnov et al., 2016). Furthermore, the average of the Precambrian dipole moment and its variability (49 $\pm 31 \mathrm{ZAm}^{2}$ according to the PINT database, Biggin et al., 2015) are indistinguishable, within uncertainties, with the 0 - 300 Ma dipole moment of $46 \pm 32 \mathrm{ZAm}^{2}$ determined by Selkin and Tauxe (2000), as well as the $49 \mathrm{ZAm}^{2}$ average dipole moment determined by Wang et al. (2015) for $0-5$ Ma. In summary, the interpretation of the paleointensity record in relation to ICN is still an unsettled and ongoing debate.

Another possible signal probe of ICN is the surface field morphology. Although most palaeomagnetic investigations suggest that the surface field has been dominated by an axial dipole for the last two billion years (Evans, 2006; Veikkolainen and Pesonen, 2014; Biggin et al., 2015), anomalies in palaeomagnetic inclinations have been attributed to a stronger octupole during the Precambrian (Kent and Smethurst, 1998; Veikkolainen and Pesonen, 2014), a potential ICN footprint (Bloxham, 2000; Heimpel and Evans, 2013).

Because the interpretation of palaeomagnetic data remains controversial, modelling the evolution of the geodynamo is particularly useful to guide the search for ICN in the palaeomagnetic record. Numerical dynamo investigations have shown that the absence of an inner core may favor unusual field structures, superimposed to the axial dipole, including the axial quadrupole producing a field localized in one hemisphere (Landeau and Aubert, 2011; Hori et al., 2014) or the axial octupole (Heimpel and Evans, 2013). However, a full modelling of the evolution of the geodynamo field morphology is still missing, and is key to testing the Geocentric Axial Dipole (GAD) hypothesis on which continental drift reconstructions rely (Evans, 2013).

Theoretical predictions of the effect of ICN on paleointensity have been obtained by coupling core energetics with dynamo scalings (Olson, 1981; Stevenson et al., 1983; Aubert et al., 2009). Results from dynamo simulations have shown that the field intensity evolves as the available power to the power $1 / 3$ (Christensen and Aubert, 2006; Christensen et al., 2009; Aubert et al., 2009). This scaling satisfactorily explains observations of magnetic field strength in planets and stars (Christensen et al., 2009). Incorporating this power-based scaling into core evolution models, Aubert et al. (2009) have shown that the cooling of the core on geological time scales provides enough power to maintain a strong dipole field (i.e. of same order as the present-day field) prior to ICN and as late as $~ 3.5 \mathrm{Ga}$, consistently with palaeomagnetic observations (Tarduno et al., 2010; Biggin et al., 2011). Aubert et al. (2009) predicted that the sharp change in available power due to ICN induced a sudden change in internal field intensity, with a field smaller by up to a factor of $\sim 4$ prior to ICN compared to just after ICN. On the basis of an empirical scaling law relating the internal and surface field intensities, Aubert et al. (2009) found that the surface intensity weakens by a factor of 2 or less near ICN relative to present-day conditions. They attributed the smaller decrease in surface intensity to the shallowing 
of the dynamo region with age, which partially compensates the overall loss of dynamo power.

In this study, we investigate the history of the geodynamo and the signature of ICN at the Earth's surface by combining the energetics of the core with dynamo simulations. Whereas Aubert et al. (2009) implement dynamo scalings into core evolution models, here, we directly apply core evolution models on dynamo simulations that model the present-day geodynamo. Because this new procedure does not rely on dynamo scalings, it can be used to investigate the evolution of any dynamo property with age, and pinpoint more precisely the effect of ICN on the morphology and intensity of the surface palaeomagnetic field. We note that modelling the long-term evolution of the reversal frequency is out of the scope of the present study. A similar general recipe was used recently by Driscoll (2016) whose results are compared to ours in Section 6.

\section{Procedure}

\subsection{General recipe}

Our procedure consists of the following steps:

1. First, we compute two core evolution models. We integrate, backward in time, the formulation by Labrosse (2003) for core thermal evolution, subject to two CMB heat flux histories, to determine the evolution, with age, of key properties that affect the geodynamo: the inner-core radius, the power available for the dynamo, and the distribution of mass anomaly fluxes (Fig. 1).

2. Second, we select numerical simulations of the present-day geodynamo, based on "Earth-like" criteria defined in Christensen et al. (2010), in order that the magnetic fields of the present-day dynamo models conform to the morphology of the present-day geomagnetic field.

3. Third, we compute ancient dynamo simulations at a sequence of epochs, including post-ICN and preICN ages. For this, we use the age-dependent properties from our core histories (step 1, Fig. 1), to compute the evolution of dimensionless geodynamo control parameters. We then apply ratios of past to present-day values of these dimensionless parameters (Fig. 3) to the present-day dynamo simulations isolated in step 2.

4. Fourth, we analyze the changes in magnetic field with age, relative to the present-day model, using output quantities defined in Section 3.6. For internal consistency, results are shown in terms of pastto-present dimensional quantity ratios.

\subsection{Core evolution model}


Given the large uncertainties in the outer core thermal conductivity and the CMB heat flux, it is not possible to uniquely determine the evolution of Earth's core. Instead, we define two end-member core evolution scenarios in order to test the sensitivity of our results to the core evolution model.

Several recent experimental and theoretical studies on core transport properties predict a rather high thermal conductivity, with an average value in the range of $100 \mathrm{~W} / \mathrm{m} / \mathrm{K}$ (de Koker et al., 2012; Pozzo et al., 2013; Gomi et al., 2013). The resulting heat flow along the adiabat is $\sim 11-15 \mathrm{TW}$, values that fall within the range of possible CMB heat flow values (Lay et al., 2008). This allows for two possible states of the present-day Earth's core: either superadiabatic or subadiabatic at the CMB.

Our core first history, hereafter referred as History 1, assumes a superadiabatic and spatially uniform CMB heat flux that is constant in time (i.e., constant with age). We choose $Q=13.1 \mathrm{TW}$ for the total CMB heat flow, equivalent to a global mean heat flux of $q=86 \mathrm{~mW} / \mathrm{m}^{2}$. This value is the time average CMB heat flux produced by the three mantle GCMs with plate motion constraints described in Olson et al. (2015), all of which generate large-scale patterns of mantle heterogeneity above the CMB that are similar to the seismically observed large low shear velocity provinces. As shown below, the core evolution model of Labrosse (2003) predicts an ICN age near $700 \mathrm{Ma}$ and yields superadiabatic thermal conditions for the last $1500 \mathrm{Ma}$ with this CMB heat flux, assuming a core thermal conductivity of $100 \mathrm{~W} / \mathrm{m} / \mathrm{K}$.

However, lower values for the CMB heat flow are possible (Lay et al., 2008) and would result in stabilizing subadiabatic conditions at the $\mathrm{CMB}$, in which case the constant CMB heat flux assumption of History 1 would preclude a convective dynamo prior to ICN. In order to model the effects of lower CMB heat flow while allowing for a convective dynamo at all times, we have constructed a second CMB heat flow history, referred to as History 2, in which the core is nearly adiabatic today and superadiabatic at pre-ICN times. For History 2, we assume $Q=12+0.003 \tau \mathrm{TW}$, where $\tau$ is age before present-day in Ma. History 2 yields approximately the same $700 \mathrm{Ma}$ inner core age.

We have applied the core evolution model formulation by Labrosse (2003) to compute the history of the core for both CMB heat flux histories, starting at the present-day and going back to 1500 Ma. As detailed in Supplementary Material, integrating backward in time yields the evolution of the inner core radius $r_{i}$, the ICB mass anomaly flux $F_{i}$, the CMB mass anomaly flux $F_{o}$ and the power generated by buoyancy forces (i.e. the power available for the dynamo)

$$
\Phi=-\int_{V_{s}} \rho \mathbf{u} \cdot \mathbf{g} \mathrm{d} V,
$$

where $V_{s}$ is the outer core volume, $\mathbf{u}$ the velocity field and $\mathbf{g}$ the gravitational field. .

Fig. 1 summarizes the important results for core Histories 1 and 2.

Multiple studies on paleo-length of day (l.o.d.) agree on a decrease of about $20 \mathrm{~s} / \mathrm{Ma}$ in l.o.d. during the last few hundreds million years, and a smaller, more uncertain, decrease during the Proterozoic (e.g. 
Williams, 2000; Denis et al., 2011). In our core histories, we consider a uniform increase in $\Omega$ with age between $0-250 \mathrm{Ma}$, amounting to a change in l.o.d. of $20 \mathrm{~s} / \mathrm{Ma}$, and a constant rotation rate for ages larger than 250 Ma.

Core History 1

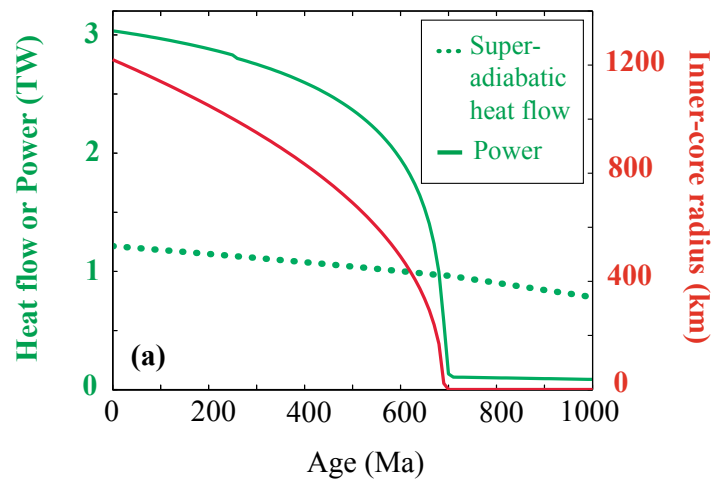

Core History 2

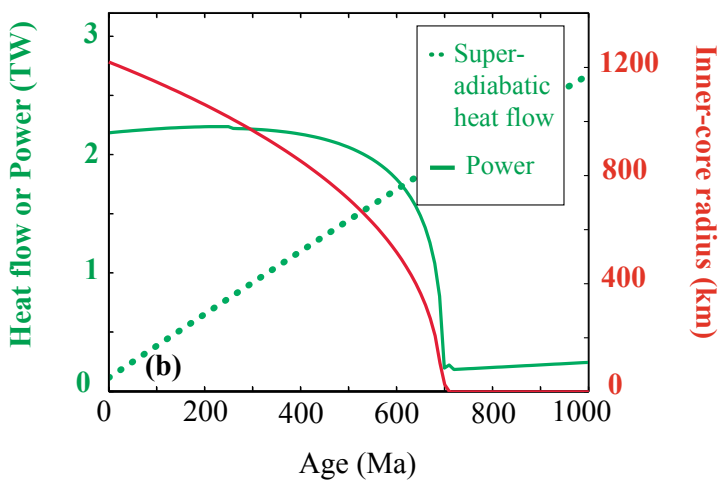

Figure 1: Thermal evolution models of the core with constant CMB heat flux (History 1, left) and CMB heat flux increasing with age (History 2, right) showing the evolution of the superadiabatic CMB heat flow $Q-Q_{a}$ (dotted green curve), power generated by the buoyancy force and available for the dynamo $\Phi$ (solid green curve) and inner-core radius $r_{i}$ (red curve), all as functions of age.

\subsection{Dynamo model and parameters}

To model thermochemical convection and dynamo action in the outer core, we follow the approach in Aubert et al. (2009). We use the numerical code PARODY-JA (Dormy et al., 1998; Aubert et al., 2008) to solve numerically the mass, momentum and energy conservation equations, together with the induction equation, for a Boussinesq, electrically conducting fluid in a self-graviting, rotating shell between radii $r_{i}$ and $r_{o}$ (representing the inner-core and core-mantle boundaries, respectively). These equations govern the evolution of the velocity field $\mathbf{u}$, the magnetic field $\mathbf{B}$ and the codensity (or density anomaly) field $C$ that accounts for thermal and compositional density anomalies (Braginsky and Roberts, 1995) such that

$$
C=\alpha \rho T+\beta \rho \xi
$$

where the temperature $T$ and the light element mass fraction $\xi$ are deviations from the mean adiabatic temperature and the mean mass fraction in the outer core, $\alpha$ and $\beta$ are the thermal and compositional expansion coefficients, and $\rho$ is the outer core density. The temperature and mass fraction fields are assumed to have the same effective diffusivitiy, $\kappa$. We impose a uniform density anomaly flux at the inner and outer 
boundaries. This amounts to imposing a mass anomaly flux

$$
F_{i}=-\int_{S_{i}} \kappa \frac{\partial C}{\partial r} \mathrm{~d} S
$$

at the inner boundary, modeling the effect of latent heat and light element release at the ICB, and a mass anomaly flux

$$
F_{o}=-\int_{S_{o}} \kappa \frac{\partial C}{\partial r} \mathrm{~d} S,
$$

at the outer boundary, representing the effect of the superadiabatic heat flux imposed by the mantle on the core. The other boundary conditions are: no-slip for the velocity and electrically insulating for the magnetic field at the inner and outer boundaries.

To model the geodynamo prior to ICN, we use full-sphere dynamo simulations where the inner-core has been completely removed. In this specific case, we impose boundary conditions at the center that force the solution and its derivatives to be regular, as detailed in Landeau (2013); this solves the problem of the singularity at the center in spherical coordinates. This full-sphere implementation in the code PARODY-JA has been validated against theoretical solutions (Landeau, 2013) and against numerical benchmarks (Marti et al., 2014).

The mean density of Earth's core evolved over geological time scales due to slow cooling of the core and light element enrichment as the inner core grows and contributes to driving core convection. Because these evolutionary effects operate on time scales much larger than the time scale relevant for core convection, they can be modeled numerically by internal buoyancy sources in a statistically stationary system (e.g. Braginsky and Roberts, 1995). Accordingly, we assume a constant and spatially uniform distribution of internal buoyancy sources, determined such that mass is conserved inside the shell (Aubert et al., 2009).

The system is then governed by six dimensionless control parameters: the ratio of inner to outer core radius, or aspect ratio,

$$
\epsilon=\frac{r_{i}}{r_{o}},
$$

the ratio of inner-boundary mass anomaly flux to total mass anomaly flux, or buoyancy source distribution,

$$
f_{i}=\frac{F_{i}}{F_{i}+F_{o}},
$$

194 the Prandtl and magnetic Prandtl numbers

$$
\operatorname{Pr}=\frac{\nu}{\kappa}, \quad \operatorname{Pm}=\frac{\nu}{\eta},
$$

where $\kappa$ and $\eta$ are diffusivities for the codensity and magnetic field, respectively, the Ekman number, which measures the ratio of viscous to rotational effects, and the modified Rayleigh number, which measures the 
vigor of convection,

$$
E=\frac{\nu}{\Omega D^{2}}, \quad R a_{Q}=\frac{g_{o} F}{4 \pi \rho \Omega^{3} D^{4}},
$$

where $g_{o}$ is gravity at the outer boundary at $r_{o}, F=F_{i}+F_{o}$ is the total mass anomaly flux, $\Omega$ the rotation rate, $D$ the shell gap between the inner and outer boundaries, and $\nu$ the viscosity of the outer core.

The Rayleigh number $R a_{Q}$ is a measure of the vigor of convection: for sufficiently supercritical convection, it is proportional to the dimensionless power density $p$ generated by the buoyancy forces, from which convection originates, such that (see Supplementary Material)

$$
p=\gamma R a_{Q},
$$

with

$$
p=\frac{\Phi / V_{s}}{\rho \Omega^{3} D^{2}},
$$

where $\Phi$ is the power generated by buoyancy forces defined by (1), $V_{s}$ the shell volume, and $\gamma$ a constant that depends on the shell geometry and the boundary conditions (Supplementary Material). Note that, by definition, the convective power $p$ measures the power available for convection and dynamo action, for which $R a_{Q}$ is only an approximate proxy that does not account for variable shell geometry or variable buoyancy source distribution. The canonical Rayleigh number $R a=g_{o} \Delta C D^{3} / \rho \nu \kappa$, where $\Delta C \equiv F / 4 \pi \kappa D$, is given by $R a=R a_{Q} E^{-3} \operatorname{Pr}^{2}$. Unlike $R a, R a_{Q}$ has the advantage of being independent of the thermal and viscous diffusivities, and is therefore the relevant parameter to study convection in the limit of low Ekman and highly supercritical conditions (Christensen and Aubert, 2006).

As shown in Section 3.2, thermochemical evolution of the core implies that the rotation rate, the innercore size, the buoyancy source distribution, and the dissipation vary over geological time scales. Therefore, four dynamo control parameters are functions of time (or age): $\epsilon, f_{i}, R a_{Q}$ and $E$.

\subsection{Present-day dynamo simulations}

To model the present-day geodynamo, we use two sets of simulation parameters (given in Table 1, Age 0 $\mathrm{Ma}$ ), one for each core history. In both simulations, the aspect ratio $\epsilon$ is chosen equal to 0.35 , its value in the present-day core. For the buoyancy source distribution $f_{i}$, we impose values equal to these predicted by our core Histories 1 and 2 to within 1\%. The Ekman number is equal to $3 \times 10^{-5}$ in both simulations, a value reasonably low compared to other published dynamo models (e.g. Driscoll, 2016; Hori et al., 2014; Heimpel and Evans, 2013) but sufficiently high to permit simulations at different ages with the two core histories.

We adjust the other parameters $\left(P r, P m, R a_{Q}\right)$ so that our dynamo simulations produce a CMB field that resembles that of the present-day core field. For this, we use criteria formulated by Christensen et al. 
(2010) to quantify the degree of resemblance of a dynamo model magnetic field with the geomagnetic field at the CMB.

Christensen et al. (2010) measure the compliance of dynamo models to the historical geomagnetic field in terms of four properties of the field up to spherical harmonic degree and order eight: the ratio of the power in the axial dipole component to that in the rest of the field, the ratio between equatorially symmetric and antisymmetric components, the ratio between zonal and non-zonal non-dipole components, and the spatial concentration of magnetic flux, all at the CMB. These properties are factored into the definition of a compliance parameter $\chi^{2}$, which varies typically between 0.3 and 20 in numerical dynamos. Excellent compliance as defined by Christensen et al. (2010) corresponds to $\chi^{2} \leq 2$. We choose present-day dynamo parameters such that the compliance parameter $\chi^{2}$ is equal to 1.4 and 0.7 (Table 1 ).

Fig. 2 illustrates morphological similarities between the geomagnetic field (Fig.2a) and the field produced by our present-day dynamo simulations (Fig.2b,c). In both cases, the radial field is dominated by an axial dipole, although significant deviations from axial and dipolar symmetries are present. For example, in the geomagnetic field and the dynamo simulations, we see strong non-axial structures that form pairs of radial field patches, antisymmetric with respect to the equator; these are the signature of columnar flows in the shell. Other non-axial field structures form single patches, such as the reversed flux patches seen in the Southern hemisphere in Fig.2a,b.

3

(a) CHAOS-4

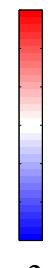

(b)

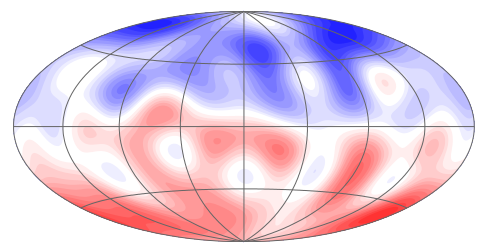

(c)

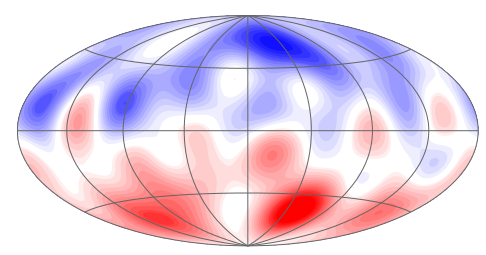

Figure 2: Radial magnetic field at the CMB up to degree eight, normalized by its root mean square value. (a) CHAOS-4 geomagnetic field model in 2010 (Olsen et al., 2014). (b,c) Snapshots from present-day dynamo simulations for History 1 and History 2, respectively (dynamo parameters given in Table 2 at age $0 \mathrm{Ma}$ ).

\subsection{Ancient dynamo simulations}

Starting from the above present-day dynamo simulations, we use our core evolution models (core Histories 1 and 2) to define a set of dynamo simulation parameters that model the geodynamo at earlier times, as illustrated in Fig.3 and described in what follows.

First, from the core histories shown in Fig.1, we compute the evolution with age of the convective power $p$ (equation (10)), the Ekman number $E$ (equation (8)), the aspect ratio $\epsilon$ (equation (5)) and the buoyancy 


\begin{tabular}{ccccccccccccc}
\hline History & Age(Ma) & $\epsilon$ & $f_{i}$ & $E$ & $R a_{Q}$ & $p$ & $\chi^{2}$ & $f_{\text {dip }}$ & $\Lambda$ & $M E / K E$ & Regime \\
\hline 1 & 0 & 0.350 & 0.93 & $3 \times 10^{-5}$ & $2.70 \times 10^{-5}$ & $8.37 \times 10^{-6}$ & 1.4 & 0.44 & 19.92 & 2.55 & $\mathrm{SF}$ & $\mathrm{St}$ \\
1 & 330 & 0.263 & 0.92 & $2.2 \times 10^{-5}$ & $1.16 \times 10^{-5}$ & $4.85 \times 10^{-6}$ & 1.5 & 0.52 & 21.31 & 3.42 & $\mathrm{SF}$ & $\mathrm{St}$ \\
1 & 540 & 0.178 & 0.90 & $1.8 \times 10^{-5}$ & $5.67 \times 10^{-6}$ & $3.02 \times 10^{-6}$ & 2.7 & 0.57 & 24.21 & 4.99 & $\mathrm{SF}$ & $\mathrm{St}$ \\
1 & 700 & 0.00 & 0.00 & $1.2 \times 10^{-5}$ & $4.32 \times 10^{-7}$ & $1.39 \times 10^{-7}$ & 7.9 & 0.58 & 11.46 & 42.04 & $\mathrm{SF}$ & $\mathrm{St}$ \\
& & 0.00 & 0.00 & $1.2 \times 10^{-5}$ & $4.32 \times 10^{-7}$ & $1.39 \times 10^{-7}$ & 7.9 & 0.58 & 0.42 & 0.65 & $\mathrm{WF}$ & $\mathrm{St}$ \\
1 & 1000 & 0.00 & 0.00 & $1.2 \times 10^{-5}$ & $3.31 \times 10^{-7}$ & $9.31 \times 10^{-8}$ & 10.9 & 0.62 & 9.68 & 50.44 & $\mathrm{SF}$ & $\mathrm{St}$ \\
& & 0.00 & 0.00 & $1.2 \times 10^{-5}$ & $3.31 \times 10^{-7}$ & $9.31 \times 10^{-8}$ & 10.9 & 0.62 & 0.33 & 0.74 & $\mathrm{WF}$ & $\mathrm{Mt}$ \\
\hline 2 & 0 & 0.350 & 0.99 & $3 \times 10^{-5}$ & $2.70 \times 10^{-5}$ & $8.63 \times 10^{-6}$ & 0.7 & 0.41 & 17.31 & 2.02 & $\mathrm{SF}$ & $\mathrm{St}$ \\
2 & 330 & 0.263 & 0.93 & $2.2 \times 10^{-5}$ & $1.38 \times 10^{-5}$ & $5.84 \times 10^{-6}$ & 1.0 & 0.49 & 21.07 & 2.75 & $\mathrm{SF}$ & $\mathrm{St}$ \\
2 & 540 & 0.178 & 0.87 & $1.74 \times 10^{-5}$ & $7.02 \times 10^{-6}$ & $3.72 \times 10^{-6}$ & 2.5 & 0.53 & 26.37 & 4.33 & $\mathrm{SF}$ & $\mathrm{St}$ \\
2 & 700 & 0.00 & 0.00 & $1.2 \times 10^{-5}$ & $7.20 \times 10^{-7}$ & $2.77 \times 10^{-7}$ & 3.8 & 0.51 & 15.38 & 30.50 & $\mathrm{SF}$ & $\mathrm{St}$ \\
& & 0.00 & 0.00 & $1.2 \times 10^{-5}$ & $7.20 \times 10^{-7}$ & $2.77 \times 10^{-7}$ & 3.8 & 0.51 & 0.51 & 0.43 & $\mathrm{WF}$ & $\mathrm{Mt}$ \\
2 & 1000 & 0.00 & 0.00 & $1.2 \times 10^{-5}$ & $9.22 \times 10^{-7}$ & $3.80 \times 10^{-7}$ & 2.1 & 0.50 & 15.21 & 21.69 & $\mathrm{SF}$ & $\mathrm{St}$ \\
& & 0.00 & 0.00 & $1.2 \times 10^{-5}$ & $9.22 \times 10^{-7}$ & $3.80 \times 10^{-7}$ & 2.1 & 0.50 & 0.71 & 0.45 & $\mathrm{WF}$ & $\mathrm{Mt}$ \\
\hline
\end{tabular}

Table 1: Parameters and diagnostics for dynamo simulations, each simulation spanning between 1 and 2 magnetic diffusion times. See Section 3 for definition of the buoyancy source distribution $f_{i}$, the Ekman number $E$, the Rayleigh number $R a_{Q}$, the convective power $p$, the relative dipole field strength $f_{\text {dip }}$, the Elsasser number $\Lambda$ and the mean ratio of magnetic to kinetic energies $M E / K E$. Two dynamo solutions are found at $700 \mathrm{Ma}$ and $1000 \mathrm{Ma}$; the last two columns characterize the regime and the stability of the dynamo solutions: 'SF', 'WF', 'St' and 'Mt' for strong-field regime, weak-field regime, stable and metastable, respectively (see section 4.1 for definitions). We follow the definition of Christensen et al. (2010) for the compliance parameter $\chi^{2}$. In all simulations, we impose $P r=1$ and $P m=2.5$. 
source distribution $f_{i}$ (equation (6)) relative to their present-day values (the subscript 0 denotes present-day values). The resulting past-to-present day ratios characterizing the evolution of the geodynamo are shown in Fig.3 and their values are given in Table 2 and Table 3.

We then select four age values (vertical dashed lines in Fig.3) such that two ancient dynamos have an inner core (330 Ma, $540 \mathrm{Ma}$ ), one ancient dynamo is located right at ICN (700 Ma) and another one is located well before the inner-core starts to nucleate (1000 Ma).

For each core history and each of these four ages, we define an ancient dynamo simulation such that $E, f_{i}$ and $\epsilon$ are equal to their values in the present-day simulation times the past-to-present day ratios $f_{i} / f_{i 0}, \epsilon / \epsilon_{i}$ and $E / E_{0}$ predicted by the core history (Table 2 and Table 3 ) to within $2 \%$ error. We then adjust the value of the Rayleigh number $R a_{Q}$ so that the ratio of past-to-present convective power in our dynamo series matches the ratio $p / p_{0}$ predicted by the core history (Table 2 and Table 3 ) to within $10 \%$ error. Note that relation (9) implies that matching $p / p_{0}$ or $R a_{Q} / R a_{Q_{0}}$ is equivalent for sufficiently supercritical conditions (satisfied only for our post-ICN simulations). The resulting control parameter values for these ancient dynamo simulations are given in Table 1.

Several reasons motivate the use of the convective power $p$ to scale our dynamo models to the Earth, instead of $R a_{Q}$. First, numerical (e.g. Christensen and Aubert, 2006; Olson and Christensen, 2006; Aubert et al., 2009; Davidson, 2013) and natural dynamos (Christensen et al., 2009) provide evidence that the convective power controls the self-sustained field intensity, which is determined through a balance between the convective power and the Ohmic dissipation. Second, practical reasons add to the above physical reason: scaling the dynamo model using past-to-present day ratios of $R a_{Q}$ would produce pre-ICN simulations located below the onset of dynamo action or even the onset of convection. Using ratios of the deviation from the onset of convection is possible, but requires knowing precisely the critical Rayleigh number for convection onset, which would add another step in the recipe.

\subsection{Output parameters}

For each present-day and ancient dynamo simulation, we compute several outputs that characterize the structure and intensity of the magnetic field. The mean internal field intensity $B_{\text {rms }}$ is measured as the time-averaged root-mean square (rms) field in the shell, such that

$$
B_{\mathrm{rms}}=\left(\frac{1}{V_{s}}\left\langle\int_{V_{s}} \mathbf{B}^{2} \mathrm{~d} V\right\rangle\right)^{1 / 2}
$$

where $\mathbf{B}$ is the dimensional magnetic field, $V_{s}$ the shell volume and the angled brackets indicate a timeaveraging operator. 


\begin{tabular}{llllll}
\hline $\begin{array}{l}\text { Age } \\
(\mathrm{Ma})\end{array}$ & $\epsilon / \epsilon_{0}$ & $f_{i} / f_{i_{0}}$ & $E / E_{0}$ & $p / p_{0}$ & $\mathrm{IC}$ \\
\hline $\mathbf{0}$ & 1.000 & 1.00 & 1.00 & 1.00 & $\mathrm{Y}$ \\
200 & 0.861 & 1.00 & 0.83 & 0.71 & $\mathrm{Y}$ \\
$\mathbf{3 3 0}$ & 0.752 & 0.99 & 0.73 & 0.57 & $\mathrm{Y}$ \\
400 & 0.683 & 0.99 & 0.69 & 0.51 & $\mathrm{Y}$ \\
$\mathbf{5 4 0}$ & 0.509 & 0.98 & 0.59 & 0.37 & $\mathrm{Y}$ \\
600 & 0.403 & 0.96 & 0.54 & 0.30 & $\mathrm{Y}$ \\
$\mathbf{7 0 0}$ & 0.000 & 0.00 & 0.39 & $1.5 \times 10^{-2}$ & $\mathrm{~N}$ \\
800 & 0.000 & 0.00 & 0.39 & $1.1 \times 10^{-2}$ & $\mathrm{~N}$ \\
$\mathbf{1 0 0 0}$ & 0.000 & 0.00 & 0.39 & $9.8 \times 10^{-3}$ & $\mathrm{~N}$ \\
1200 & 0.000 & 0.00 & 0.39 & $8.3 \times 10^{-3}$ & $\mathrm{~N}$ \\
1400 & 0.000 & 0.00 & 0.39 & $6.8 \times 10^{-3}$ & $\mathrm{~N}$ \\
\hline
\end{tabular}

Table 2: Evolution of dynamo parameters as predicted by core History 1 (see Section 3 for details). The subscript 0 denotes present-day parameter values. The present-day values of $f_{i}, \epsilon, E$ and $p$ are equal to $0.94,0.35,2.71 \times 10^{-15}$ and $8.32 \times 10^{-13}$, respectively, in core History 1 . Bold characters indicate age values at which we compute dynamo simulations. The last column indicates the presence of an inner core: ' $\mathrm{Y}$ ' for ages with an active inner core and ' $\mathrm{N}$ ' for ages without an active inner core.

\begin{tabular}{llllll}
\hline Age & $\epsilon / \epsilon_{0}$ & $f_{i} / f_{i_{0}}$ & $E / E_{0}$ & $p / p_{0}$ & $\mathrm{IC}$ \\
$(\mathrm{Ma})$ & & & & & \\
\hline $\mathbf{0}$ & 1.000 & 1.00 & 1.00 & 1.00 & $\mathrm{Y}$ \\
200 & 0.870 & 0.97 & 0.84 & 0.77 & $\mathrm{Y}$ \\
$\mathbf{3 3 0}$ & 0.766 & 0.95 & 0.74 & 0.66 & $\mathrm{Y}$ \\
400 & 0.700 & 0.94 & 0.70 & 0.60 & $\mathrm{Y}$ \\
$\mathbf{5 4 0}$ & 0.528 & 0.90 & 0.60 & 0.47 & $\mathrm{Y}$ \\
600 & 0.423 & 0.88 & 0.55 & 0.39 & $\mathrm{Y}$ \\
$\mathbf{7 0 0}$ & 0.022 & 0.00 & 0.42 & $3.1 \times 10^{-2}$ & $\mathrm{~N}$ \\
800 & 0.000 & 0.00 & 0.39 & $3.1 \times 10^{-2}$ & $\mathrm{~N}$ \\
$\mathbf{1 0 0 0}$ & 0.000 & 0.00 & 0.39 & $3.8 \times 10^{-2}$ & $\mathrm{~N}$ \\
1200 & 0.000 & 0.00 & 0.39 & $4.4 \times 10^{-2}$ & $\mathrm{~N}$ \\
1400 & 0.000 & 0.00 & 0.39 & $5.1 \times 10^{-2}$ & $\mathrm{~N}$ \\
\hline
\end{tabular}

Table 3: Same as for Fig.2 but for core History 2. The present-day values of $f_{i}, \epsilon, E$ and $p$ are equal to $0.99,0.35,2.71 \times 10^{-15}$ and $7.29 \times 10^{-13}$, respectively, in core History 2 . 
Core History 1

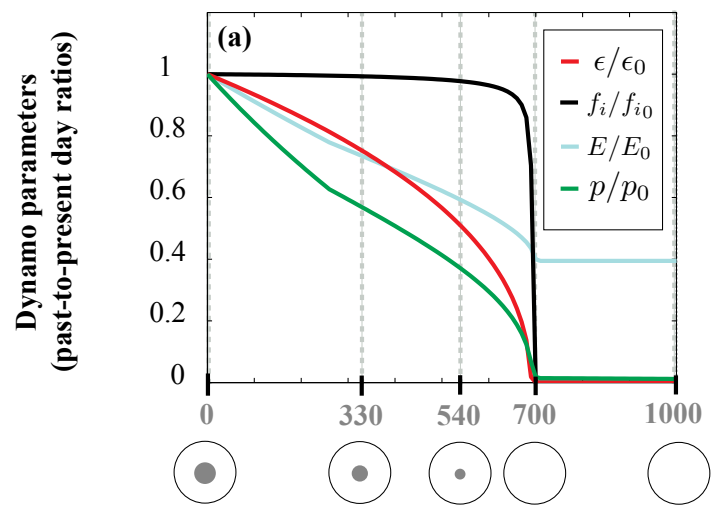

Age (Ma)
Core History 2

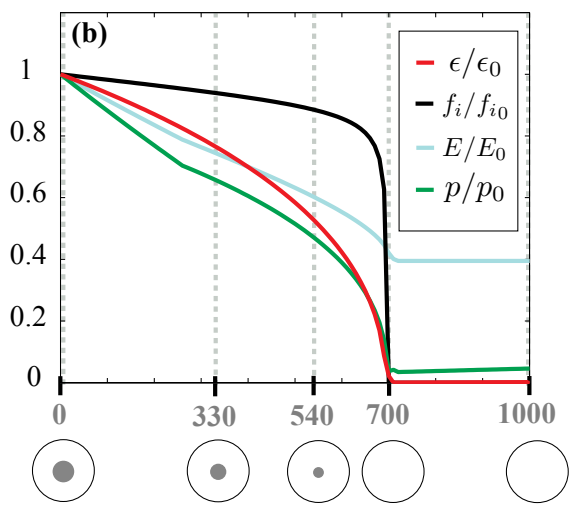

Age (Ma)

Figure 3: Evolution of geodynamo parameters (aspect ratio $\epsilon$, Ekman number $E$, convective power $p$ and buoyancy source distribution $f_{i}$ ) relative to their present-day values (denoted by the subscript 0 ), as deduced from core History 1 (left) and core History 2 (right). Grey vertical dashed lines locate ages where dynamo simulations are computed; schematic grey icons show the corresponding core structure, filled black circles representing the inner core.

The surface field intensity is measured through the time-averaged dipole moment $M$ defined as follows:

$$
M=\frac{4 \pi r_{o}{ }^{3}}{\sqrt{2} \mu} B_{\mathrm{dip}},
$$

where $\mu$ is the magnetic permeability, and $B_{\text {dip }}$ the time-averaged rms dipole field intensity at $r_{o}$.

The relative dipole field strength $f_{\text {dip }}$ is measured as the time-average ratio of the rms dipole field intensity to the rms of the total field on the outer shell boundary. We also compute time-averaged ratios of Schmidt-normalized Gauss coefficients: the ratio $G_{2}$ of axial quadrupole to axial dipole and the ratio $G_{3}$ of axial octupole to axial dipole. We compute the Elsasser number $\Lambda=B_{\mathrm{rms}}{ }^{2} / \rho \mu \eta \Omega$, which measures the relative strength of the Lorentz and Coriolis forces, and the time-averaged ratio of magnetic to kinetic energy $M E / K E$.

The quantities $f_{\text {dip }}, M E / K E, G_{2}$ and $G_{3}$ are dimensionless ratios of output quantities; therefore, no scaling is needed to extrapolate the results from dynamo simulations to the geodynamo. This is not true for the field intensities or the dipole moment whose extrapolation to the Earth implies the choice of a scaling procedure. To avoid any arbitrary nondimensionalization of the magnetic field, we show the results in terms of ratios of past-to-present day dimensional values. With this choice, the results are independent of the characteristic time scale and length scale chosen to define the control dynamo parameters (5)-(8). 


\section{Evolution dynamo regime and magnetic field morphology with age}

\subsection{Geodynamo regimes: strong-field versus weak-field states}

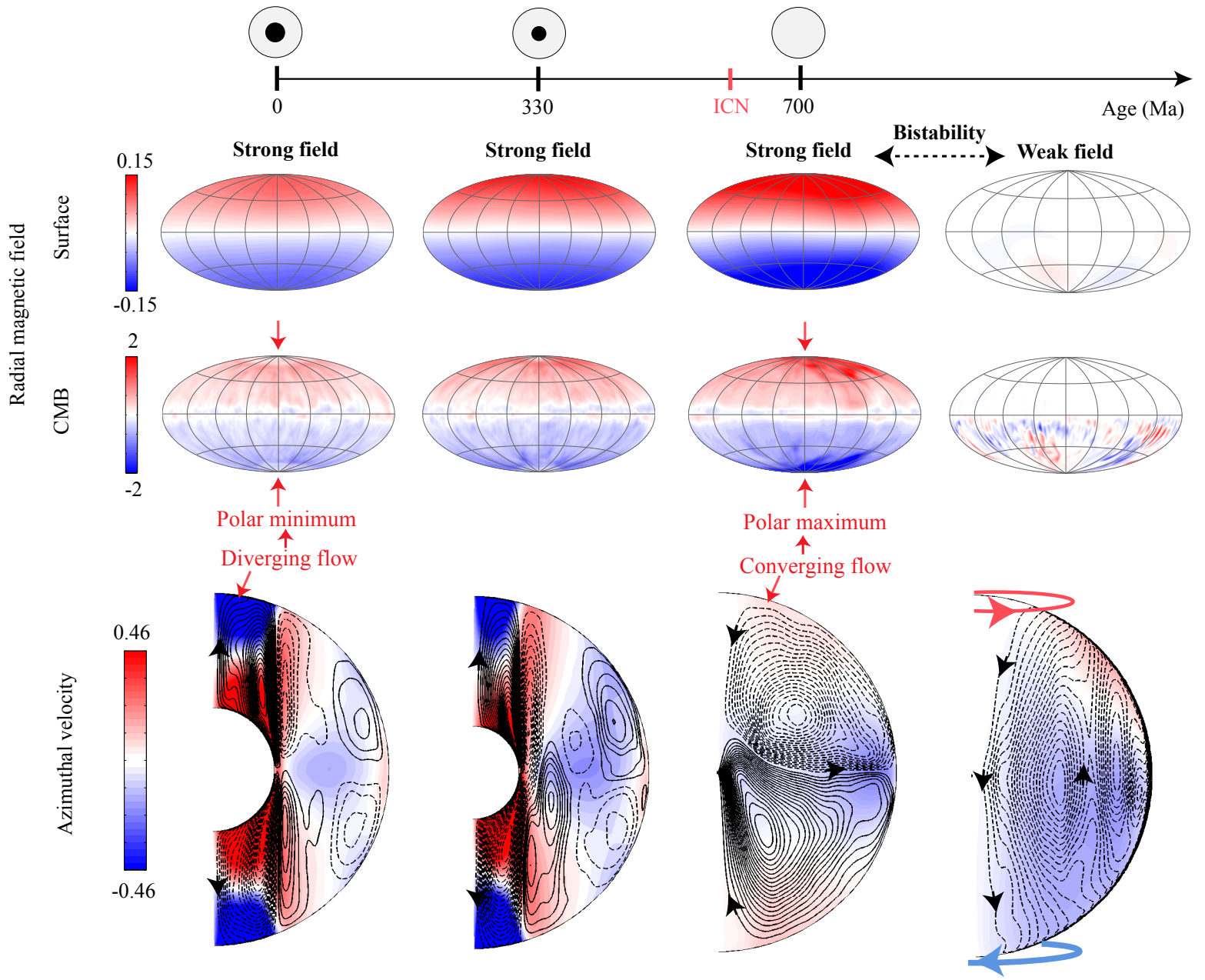

(a)

(c)

(d)

Figure 4: Dynamo structures using constant CMB heat flow History 1 at 0 Ma (first column), 330 Ma (second column) and $700 \mathrm{Ma}$ (third and fourth columns). (a) Age in Ma with corresponding core structure, the black filled circle representing the inner core. (b,c) Radial magnetic field at the surface of the Earth, assuming a $2890 \mathrm{~km}$ thick electrically insulating mantle (b) and at the CMB (c), normalized by the present-day rms field intensity at the CMB (Hammer projections, the rotation axis is vertical). Time-averaged magnetic field in the first three columns (strong-field dynamo); snapshots in the fourth column (weak-field dynamo). (d) Meridional cuts of the time-averaged azimuthal velocity, normalized by the present-day rms velocity inside the shell. Contours show streamlines of the meridional circulation which rotates clockwise (solid lines) or anticlockwise (dashed lines).

Fig.4 shows our results for the evolution of the magnetic field structure as a function of age at the CMB (Fig.4c) and at the Earth's surface (Fig.4b), as obtained in dynamo simulations defined using core History 1. 
At all ages when an inner core is present, the CMB field is dominated by an axial dipole (first and second panels in Fig.4c and $f_{\text {dip }}$ in Table 1). As a result, the surface field is also dominated by an axial dipole (Fig.4b, first and second panels), with a mean axial dipole Gauss coefficient always larger by a factor 20 or more than other mean Gauss coefficients. Note that $f_{\text {dip }}$ increases slightly with age (Table 1), meaning that the dipolarity of the field increases back in time. Because the magnetic field is dipole dominated, it is mainly symmetric with respect to the equator, showing no particular localization in one hemisphere. Our post-ICN dynamo simulations are all characterized by a ratio of magnetic to kinetic energy larger than 1 and an Elsasser number larger than 10 (Table 1); they are therefore in the so-called strong-field regime (e.g. Roberts, 1988). Such a dipole-dominated regime has been extensively studied and results from field generation by drifting columnar flows (e.g. Olson et al., 1999; Aubert et al., 2008), mainly symmetric with respect to the equator (first two panels in Fig.4d).

The above dipolar strong-field state is also stable prior to ICN (Table 1 and third column in Fig.4). However, prior to ICN, an additional solution emerges and is shown in the fourth column of Fig.4. This second dynamo solution is in a weak-field regime where both the ratio of magnetic to kinetic energy and the Elsasser number are smaller than 1 (Table 1). The weak-field dynamo state is characterized by a multipolar field (as shown by the value of $f_{d i p}$ in Table 1), which reverses frequently. We record an average of 49 apparent polarity reversals per magnetic diffusion time in the weak-field state, whereas the corresponding strong-field dynamo does not reverse in one or two diffusion times. The weak field is also strongly hemispherical with a magnetic field 6 times more intense in one hemisphere than in the other at the CMB and 2 times at the Earth's surface.

This hemispherical magnetic field is associated with a strongly asymmetric time-averaged flow, shown in the fourth panel of Fig.4d, which contrasts with the equatorially symmetric flow in the strong-field regime (third panel in Fig.4d). To characterize the symmetry of the time-averaged flow we measure the ratio of the kinetic energy $K_{a}$ of the antisymmetric time-averaged flow to the total kinetic energy $K$ of the timeaveraged flow. At 700 Ma with core History 1 , the ratio $K_{a} / K$ is equal to 0.25 in the weak-field state whereas it is equal to 0.06 in the strong-field state. The asymmetric time-averaged flow in the weak-field regime is characterized by a large-scale meridional cell that goes through the center of the core and two patches of azimuthal velocity forming two large-scale vortices of opposite sign, one in each hemisphere (blue and red arrows in the fourth panel of Fig.4d). This type of convection has been named EAA (equatoriallyantisymmetric and axisymmetric) convection and its dynamics has been described in detail in Landeau and Aubert (2011). Because it relies on the possibility of axial flow going through the center, this type of convection is suppressed by an active inner core, and is therefore not observed after ICN.

When bistability occurs prior to ICN, the final state of the dynamo depends on initial conditions: an 
infinitesimally small initial magnetic field leads to a weak-field dynamo whereas a strong enough initial magnetic field leads to a strong-field dynamo. Bistability between the strong-field and weak-field states is marginal. We find that the weak-field regime is stable between $p=9.31 \times 10^{-8}$ and $p=2.77 \times 10^{-7}$ at the Ekman number of our pre-ICN simulations (Table 1), a very narrow stability domain considering that the convective power varies by about two orders of magnitude between 0 Ma and 1000 Ma in our core evolution models. As a result, whether or not the dynamo reaches the weak-field regime is sensitive to the core evolution scenario; this is illustrated by our core History 2 which never reaches the stability domain of the weak-field regime and remains in the strong-field dipolar regime prior and after ICN (Table 1).

Despite this narrow stability domain, we find that, at all pre-ICN ages where it is not stable, the weak-field regime is metastable: starting from an infinitesimally small magnetic field, the weak-field state is maintained for 0.2 to 1 magnetic diffusion times before finally transitioning to the strong-field dipolar state. We do not observe any metastability of the weak-field state after ICN.

\subsection{Evolution of magnetic field morphology in the strong-field regime}

Our core History 2 predicts that the dynamo might have spent the last 2 Ga exclusively in a strong-field dipolar regime. In this section, we investigate the effect of ICN on the magnetic field morphology within the strong-field dynamo regime.

\subsubsection{Surface field morphology: octupole strengthening with age}

Although the strong-field dynamo remains dominated by an axial dipole at all ages (Fig.4bc), secondary morphological changes at Earth's surface can be quantified by computing the first Gauss coefficients of the surface field. Fig.5 shows the evolution of the Gauss coefficient ratios $G_{2}$ and $G_{3}$ of the time-averaged field in the strong-field dynamo state. The ratio $G_{2}$ of the axial quadrupole Gauss coefficient to the axial dipole Gauss coefficient shows no consistent trend with age, fluctuating within the $1 \%$ range (Fig.5a). In contrast, the axial octupole coefficient ratio $G_{3}$ progressively increases with age as the inner core vanishes; this is consistently found for both core evolution scenarios, as shown in Fig.5a. Starting from negative present-day values, $G_{3}$ changes sign within the last $330 \mathrm{Ma}$ and reaches positive values of about $4-5 \%$ around ICN. For ages larger than ICN, $G_{3}$ decreases with age for core History 2, whereas it remains approximately constant for core History 1.

\subsubsection{Dynamical origin of the octupole strengthening}

In what follows, we interpret the increase in octupole strength as a result of a purely geometric effect as the inner core shrinks. 
After ICN (first and second panels in Fig.4d), the strong buoyancy flux at the ICB induces strong upwellings that rise vertically to the poles below the $\mathrm{CMB}$, feeding two meridional cells that are associated with anticyclonic polar vortices below the CMB. These polar flows have been documented in previous studies (Olson and Aurnou, 1999; Sreenivasan and Jones, 2005). As a result of the Taylor-Proudman constraint, which forces the flow to be two-dimensional in rapidly rotating systems, the width of the polar meridional cells in the first and second panels of Fig.4d is equal to the width of the tangent cylinder (an imaginary cylinder tangent to the inner core and aligned with the rotation axis).

In constrast, prior to ICN, we observe downwellings near the poles below the CMB: convection, now driven at the CMB by core cooling alone, forms downwellings that detach from the CMB and plunge along the rotation axis (third panel in Fig.4d). Without an inner core and therefore a tangent cylinder, polar flows become coupled with the rest of the sphere: the polar downwellings merge with two meridional cells that span the entire outer core.

To understand how the above changes in polar flows affect the magnetic field morphology at the CMB (three first panels in Fig.4c), it is important to recall that convergent flows below the CMB tend to collect field lines and amplify the magnetic field by stretching whereas divergent flows tend to advect the field lines away, producing a local minimum in field intensity, as described in previous studies (e.g. Aubert et al., 2008). In the presence of an inner core, the flow upwellings induce a divergent flow below the poles (two first panels in Fig.4d) and, therefore, produce a local minimum in magnetic field intensity there (two first panels in Fig.4c). As a result, the width of the polar upwellings, dictated by the width of the tangent cylinder, controls the size of the polar minimum in magnetic intensity at the CMB. This explains why the polar minimum in magnetic intensity gets narrower with age as the inner core shrinks (Fig.4c, two first panels). In contrast, prior to ICN, the polar downwellings induce a convergent polar flow below the CMB which amplifies magnetic field there, and causes a polar maximum in radial field intensity at the CMB (Fig.4c, third panel).

Finally, the above transition from polar minimum in magnetic field intensity to polar maximum at the $\mathrm{CMB}$ as the inner core vanishes is directly responsible for the increase in axial octupole component with age at the Earth's surface. Given the structure of the axial dipole and the axial octupole (Fig.5b), a wide polar minimum in intensity at the CMB, as found in the present-day model (Fig.4c), requires an axial octupole of opposite sign compared to the axial dipole. This explains the negative $G_{3}$ value found in our present-day dynamos (Fig.5a). Conversely, obtaining a local polar maximum prior to ICN (700 Ma in Fig.4c), requires the axial octupole to be of same sign as the axial dipole (Fig.5b), corresponding to a positive $G_{3}$ value at Earth's surface (Fig.5a). This also explains why the increase in octupole is progressive from present-day to ICN (Fig.5a): as the tangent cylinder shrinks, the width width of the polar intensity minimum decreases, implying greater contribution from higher harmonics of the field and relatively less contribution from the 
(a)

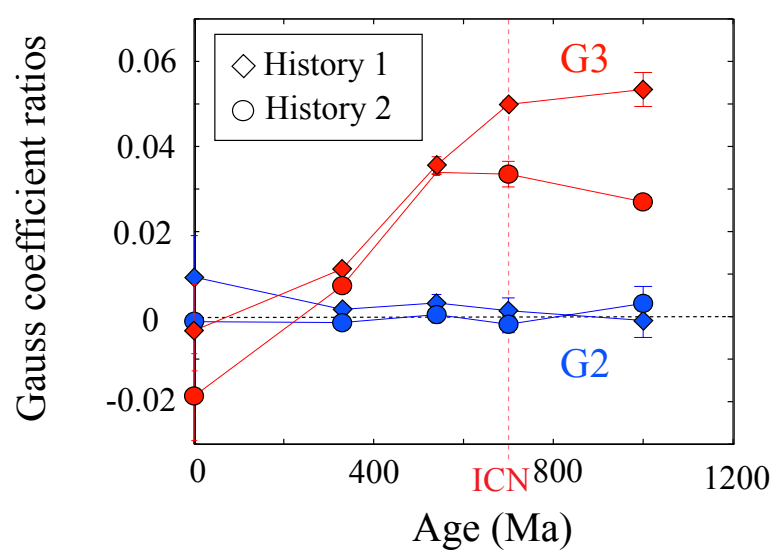

(b)
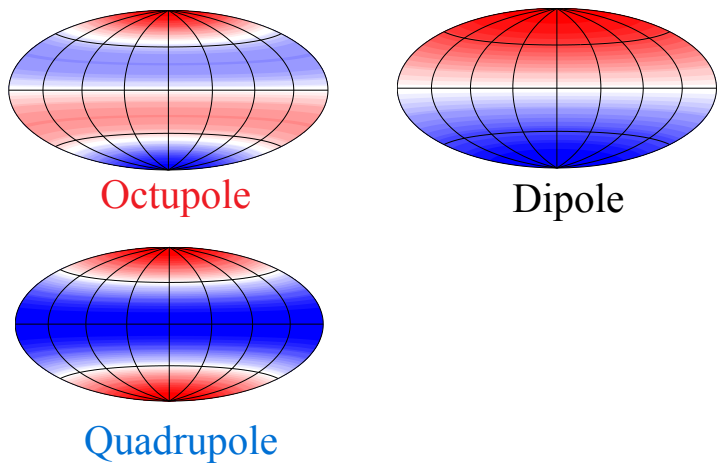

Dipole

Figure 5: (a) Evolution of mean Gauss coefficient ratios as a function of age obtained in dynamo simulations in the strong-field dynamo regime defined using core History 1 (diamonds) and core History 2 (circles). Red symbols: mean ratio $G_{3}$ of the axial octupole coefficient to the axial dipole coefficient. Blue symbols: mean ratio $G_{2}$ of the axial quadrupole coefficient to the axial dipole coefficient. Vertical bars denote errors on mean Gauss coefficient ratios. (b) Radial magnetic field structure of the axial dipole, the axial octupole and the axial quadrupole in Hammer projection (the rotation axis is vertical).

\section{Evolution of field intensity with age}

\subsection{Weak-field dynamo regime}

The red squares in Fig.6 show the evolution of the dipole moment in our dynamo simulations, normalized to the present-day. The most striking feature is the difference in dipole moment between the strong-field and weak-field dynamo states: the dipole moment in the weak-field state is up to 70 times weaker than its value in the strong-field state at a given age. Low dipole intensity in the weak-field regime results partly from the magnetic field being multipolar, and partly from the magnetic field intensity inside the core being weaker than in the strong-field regime (blue squares in Fig.6).

\subsection{Strong-field dynamo regime}

\subsubsection{Internal versus surface field intensity}

Focusing only on the strong-field dynamo regime in Fig.6, the dipole moment increases monotically with age by a factor 1.6 (core History 1 ) or 2 (core History 2 ) until the inner core vanishes. ICN has only a very minor effect on surface field intensity, with an increase in dipole moment by a factor 1.2 at most between 540 Ma and ICN at 700 Ma. For ages larger than $700 \mathrm{Ma}$, in the absence of an inner core, the dipole moment 
shows no significant variation given the uncertainties. This increase in dipole intensity with age is unexpected and counter-intuitive, in light of the fact that the convective power $p$ decreases with age, droping by more than one order of magnitude between $540 \mathrm{Ma}$ and $700 \mathrm{Ma}$ (Tables 2 and 3, Fig.3).

Numerical dynamo investigations (Christensen and Aubert, 2006; Christensen et al., 2009; Aubert et al., 2009) find that the rms internal magnetic field strength is controlled by the convective power available to balance Ohmic dissipation, provided that the field is dipole-dominated, such that

$$
B_{\mathrm{rms}} \propto f_{\mathrm{ohm}}^{1 / 2} p^{1 / 3}(\rho \mu)^{1 / 2} \Omega D,
$$

where $f_{\text {ohm }}$ the Ohmic dissipation fraction of the convective power (as introduced in Christensen and Aubert, 2006). Scaling (13) can be derived from dimensional analysis, assuming that the field strength is independent of the rotation rate $\Omega$ ( $p$ varies as $\Omega^{-3}$ by definition) (Davidson, 2013), and it successfully explains field strength observations for planetary and stellar dynamos (Christensen et al., 2009). Equation (13) can be converted into past-to-present day ratios:

$$
\frac{B_{\mathrm{rms}}}{B_{\mathrm{rms} 0}}\left(\frac{f_{\mathrm{ohm} 0}}{f_{\mathrm{ohm}}}\right)^{1 / 2}=\left(\frac{p}{p_{0}}\right)^{1 / 3} \frac{D \Omega}{D_{0} \Omega_{0}} .
$$

The blue curves in Fig. 6 show the evolution of the field intensity as predicted by scaling (14), taking $f_{\text {ohm }}=1$ at all times, as usually assumed for Earth's core (Christensen and Aubert, 2006). Acording to scaling (13), magnetic field intensity is positively correlated with the convective power, therefore, the sharp decrease in convective power near ICN (between $540 \mathrm{Ma}$ and $700 \mathrm{Ma}$ in Fig.3) is expected to cause a decrease in field intensity (blue curves in Fig.6). The increase in dipole moment from present-day to ICN, shown in Fig.6, is at variance with this prediction.

The above discrepancy between power-based arguments and the evolution of the dipole moment, does not, however, originate from an inconsistency with power-based scalings: the internal field intensity in our dynamo simulations (blue symbols in Fig.6) does follow scaling (14), with a clear decrease between 540 Ma and $700 \mathrm{Ma}$ as the inner core vanishes, trending opposite to the dipole moment. In Supplementary Material, we provide further confirmation of the consistency between our simulations and power-based scaling (13).

\subsubsection{Dynamical origin of dipole intensity increase}

The discrepancy between the evolution of the internal field and the dipole field at the surface can be attributed to the depth of the dynamo region, which varies with the buoyancy source distribution $f_{i}$ as the core evolves. This is illustrated in Fig.7, which compares magnetic energy densities before and after ICN. When the inner core is present, the convective power driving dynamo action mainly comes from the buoyancy flux at the ICB (i.e. $f_{i} \approx 1$ ), through light element and latent heat release. In this case, we obtain deeplyseated dynamos where magnetic energy density is high close to the ICB. In contrast, in the absence of an 


\section{(a) Core History 1}

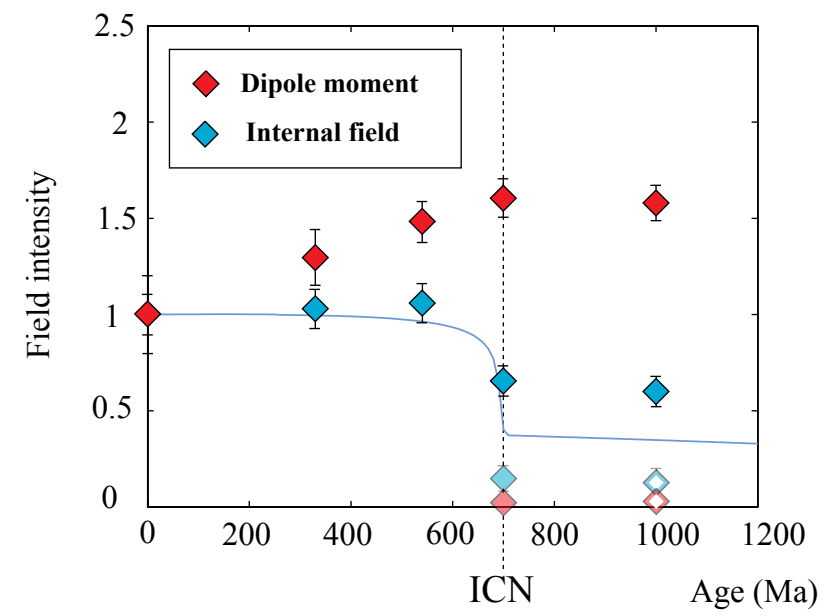

(b) Core History 2

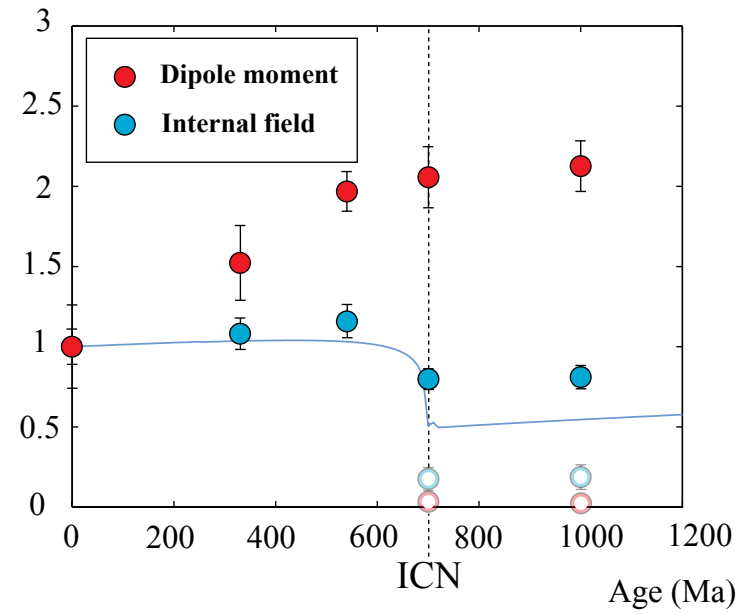

Figure 6: Evolution of magnetic field intensity with age from dynamo simulations using core History 1 (a) and core History 2 (b). Results are shown in terms of past to present-day ratios of dimensional quantities. Red symbols denote dipole moment $M / M_{0}$, blue symbols denote rms magnetic field intensity inside the outer core $B_{\mathrm{rms}} / B_{\mathrm{rms} 0}$ and blue solid curves show the internal field intensity predicted by the power-based scaling (14). Following Christensen and Aubert (2006), internal and dipole field intensities have been corrected for ohmic dissipation fraction $f_{\mathrm{ohm}}$. Saturated colors denote strong-field dynamo states and lighter colors weak-field states. Full symbols denote stable solutions; empty symbols denote unstable weak-field solutions that can be maintained for 0.2 to 1.3 magnetic diffusion times before transitioning to a stable strong-field state. 
inner core, when the destabilizing buoyancy flux comes from the CMB (i.e. $f_{i}=0$ ), the dynamo is shallower, with greater magnetic energy density just below the CMB.

Because the dynamo region becomes shallower with age, a larger fraction of magnetic energy escapes the core, which causes the increase in surface to internal intensity ratio seen in Fig.6. In addition, the magnetic field is dominated by larger scales prior to ICN than at present day (Fig.7 and $f_{\text {dip }}$ in Table 1), which contributes slightly to the increase in surface to internal intensity rato. The shallowing of the dynamo region from present-day to ICN, combined with the increase in dipolarity of the field, more than compensate for the decrease in power, allowing the dipole moment to increase with age.

$0 \mathrm{Ma}$

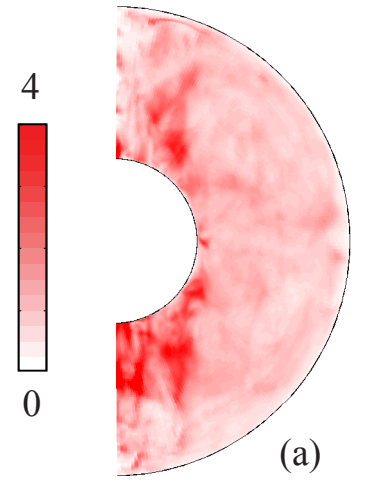

(a)

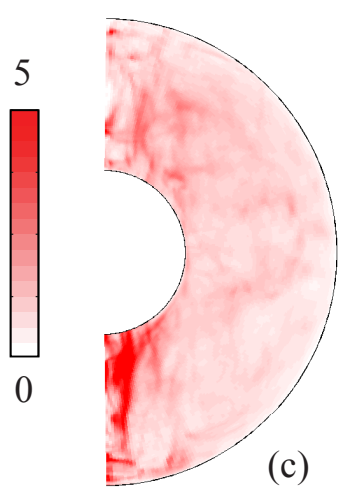

$700 \mathrm{Ma}$

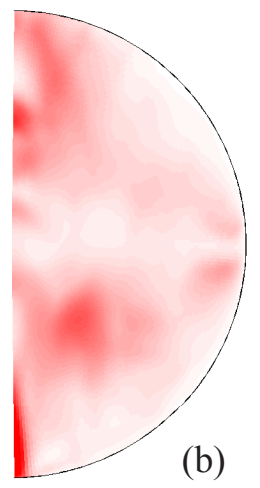

(b)

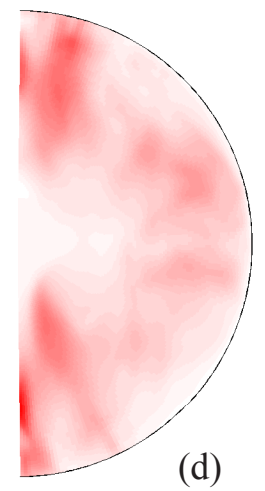

Figure 7: Meridional cuts of the axial magnetic energy density (snapshots normalized by present-day mean energy density) in present-day (0Ma, left) and pre-ICN (700 Ma, right) strong-field dynamo simulations defined using History 1 (a,b) and History $2(\mathrm{c}, \mathrm{d})$. 


\section{Discussion and conclusion}

Our results first indicate that dipole-dominated, strong-field dynamos are stable at any age. Prior to ICN, though, bistability is observed, indicating that a second regime with a weak, multipolar dynamo field is possible. Our results largely confirm recent findings of Driscoll (2016), i.e. the possibility of weak-field dynamos prior to ICN, characterized by an Elsasser number smaller than 1 and a ratio of magnetic to kinetic energy on the order of 1 .

In the weak-field dynamo state, the magnetic field is multipolar, with frequent polarity reversals and a dipole intensity up to 70 times weaker than in the strong-field dynamo state, and which does not conform to standard dynamo scalings (Christensen and Aubert, 2006; Aubert et al., 2009). A transient weak-field geodynamo state at or prior to ICN may therefore explain anomalies in the palaeomagnetic record; this catastrophic scenario has been suggested by Driscoll (2016). Because the weak-field dynamo state exists only prior to ICN, robust anomalies in the palaeomagnetic record could provide an upper bound on the age of the inner core.

No bistability was reported in Driscoll (2016). This leads the author to argue for the above catastrophic scenario and conclude that the weak-field dynamo state is an obligatory passage close to ICN, with palaeomagnetic anomalies as markers of ICN. Because our present results clearly indicate bistability, our conclusions differ from those of Driscoll (2016): we argue in favor of a simpler, uniformitarian scenario in which the geodynamo spent the last $2 \mathrm{Ga}$, both prior and after ICN, exclusively in a strong-field dipole-dominated regime. We note that, contrary to our pre-ICN simulations where the inner core has been properly removed, a small inner core is retained in Driscoll (2016) for numerical reasons. This is however unlikely to explain differences in stability behavior since retaining a small, passive inner core little affects the solution (Landeau and Aubert, 2011).

A major shortcoming of the catastrophic scenario comes from the narrow stability region of the weak-field dynamo state, which only permits for a threefold variation in convective power, a negligible range relative to the two orders of magnitude over which power can vary during the past billion years (Figure 3). In addition, in our bistable dynamos, we did not observe spontaneous transitions from a strong to a weak-field state. Because palaeomagnetic observations require the geodynamo to be in a strong-field dipolar regime at some time prior to ICN (Evans, 2006; Biggin et al., 2015; Smirnov et al., 2011), a significant external perturbation of the dynamo would be needed in order to access the weak-field regime. Variations in the CMB heat flow could for instance temporarily shut down the dynamo and restart it in the weak-field regime, but this would require a fine-tuned thermal evolution scenario.

In contrast, the uniformitarian scenario is insensitive to the details of the core thermal evolution, as illustrated by our two core evolution scenarios in the strong-field state. We note for instance that, although 
we do not explicitly consider the low-conductivity value recently suggested by Konôpková et al. (2016) in our core evolution models, we do not expect such a scenario to alter our main conclusions. Indeed, lowering the adiabatic heat flow in History 1, while maintaining the same constant and uniform CMB superadiabatic heat flow, results in a core evolution identical to History 1, but with a drop in power near ICN smaller by a factor $\sim 7$. Therefore, a low-conductivity scenario would mainly reduce the amplitude of ICN footprints, without affecting the overall dynamo behavior.

The uniformitarian scenario has other strengths. It is consistent with the quasi-stationary reversal state of the geodynamo inferred from polarity ratios in the palaeomagnetic record during the last two billion years (Driscoll and Evans, 2016). A dipole-dominated geodynamo during the last 2 billion years is consistent with palaeomagnetic observations, including inclination frequency distribution (Veikkolainen and Pesonen, 2014), correlation between paleointensity and inclination (Biggin et al., 2015), latitudinal dependence of paleosecular variation (Biggin et al., 2008; Smirnov et al., 2011) and paleoclimatic indicators of latitude (Evans, 2006), and it would furthermore support the Geocentric Axial Dipole hypothesis on which continental drift reconstruction relies (Evans, 2013). Lastly, the increase in field dipolarity with age suggested by our strong field dynamos is consistent with paleosecular variations (Smirnov et al., 2011).

Long-term trends in paleointensity have long been suspected to bear ICN footprints (e.g. Hale, 1987; Valet et al., 2014; Biggin et al., 2015) but no uncontroversial trend has been isolated so far (Smirnov et al., 2016). Our uniformitarian scenario rationalises this lack of trend. The surface magnetic field intensity from the strong-field dynamo models slowly increases with age from present-day to ICN, with depth changes of the dynamo region compensating the decrease in internal field predicted by dynamo scaling laws (Stevenson et al., 1983; Olson, 1981; Aubert et al., 2009). We note that the slow intensity increase of the surface field predicted by our results applies only to the time that includes ICN, i.e. the last $\sim 2$ Ga, and cannot be extended to older times.

Rather than paleointensity, our results point towards a long-term evolution in the amplitude of the axial octupole as a proxy for inner-core growth. We find that, in the strong-field dipole-dominated regime, the octupole is stronger prior to ICN than at present-day, consistent with previous dynamo simulations (Heimpel and Evans, 2013). Our results suggest that ICN is marked at the Earth's surface by a change in slope in the time-evolution of the Gauss coefficient ratio $G_{3}$ associated with the axial octupole: we find that $G_{3}$ increases monotonically from negative values at present day to positive values of the order of $5 \%$ at ICN, and then remains constant (or even decreases) for ages larger than ICN. This increase in octupole strength results from changes in polar flows caused by a purely geometric effect as the inner core shrinks with age.

Analysing anomalies in palaeomagnetic inclinations, Kent and Smethurst (1998) have argued for a relatively strong octupole $\left(G_{3}\right.$ up to $\left.\approx 25 \%\right)$ in the Precambrian. A more recent investigation (Veikkolainen and 
Pesonen, 2014) suggests a smaller value up to $8 \%$, still significantly larger than the value $1.1 \% \pm 1.2 \%$ for the last five million years $G_{3}$ (McElhinny, 2004). Our results suggest that the increase in $G_{3}$ may be explained by the shrinking of the inner core, without requiring any heat flux heterogeneities at the CMB (Bloxham, 2000). An important question that needs to be answered by future investigations is whether a change in slope in $G_{3}$ can be resolved in the palaeomagnetic record within the last $1-1.5$ billion years. In our view, this would provide a robust palaeomagnetic signature for ICN.

Finally, the weak-field dynamo states that pertain to pre-ICN conditions have some unexpected fluid mechanical properties, including a multipolar and hemispherical magnetic field stable at intermediate rather than high forcing, at variance with previous studies (Landeau and Aubert, 2011; Christensen and Aubert, 2006). Such pecularities of the weak-field regime obtained here at low Ekman number call for additional fluid mechanical analyses applied to planetary dynamos with passive or missing inner core.

Acknowledgements: This work was supported by the National Science Foundation through grant EAR1135382 from the Frontiers in Earth System Dynamics program. This work was granted access to the HPC resources of S-CAPAD, IPGP, France, and to the HPC resources of TGCC, CINES and IDRIS under the allocation 2016-042122 made by GENCI.

Author contributions: ML, JA, and PO jointly designed the study, JA and ML conducted the numerical simulations, ML and PO calculated the thermal evolution models, ML, JA, and PO contributed to the analysis and interpretation of data; ML, JA and PO wrote the manuscript and approved the submitted version.

\section{References}

Aubert, J., Aurnou, J., Wicht, J., 2008. The magnetic structure of convection-driven numerical dynamos. Geophys. J. Int. 172, 945-956.

Aubert, J., Labrosse, S., Poitou, C., 2009. Modelling the palaeo-evolution of the geodynamo. Geophys. J. Int. 179 (3), 1414-1428.

Badro, J., Siebert, J., Nimmo, F., 2016. An early geodynamo driven by exsolution of mantle components from earth's core. Nature 536 (7616), 326-328.

Biggin, A. J., de Wit, M. J., Langereis, C. G., Zegers, T. E., Voute, S., Dekkers, M. J., Drost, K., 2011. Palaeomagnetism of Archaean rocks of the Onverwacht Group, Barberton Greenstone Belt (southern Africa): 
Evidence for a stable and potentially reversing geomagnetic field at ca. 3.5 Ga. Earth Planet. Sci. Lett. $302(3-4), 314-328$.

Biggin, A. J., Piispa, E. J., Pesonen, L. J., Holme, R., Paterson, G. A., Veikkolainen, T., Tauxe, L., 2015. Palaeomagnetic field intensity variations suggest mesoproterozoic inner-core nucleation. Nature 526 (7572), $245-248$.

Biggin, A. J., Strik, G. H. M. A., Langereis, C. G., 2008. Evidence for a very-long-term trend in geomagnetic secular variation. Nature Geosci. 1 (6), 395-398.

Bloxham, J., 2000. Sensitivity of the geomagnetic axial dipole to thermal core-mantle interactions. Nature 405 (6782), 63-65.

Braginsky, S., Roberts, P., 1995. Equations governing convection in Earth's core and the geodynamo. Geophys. Astrophys. Fluid Dyn. 79 (1-4), 1-97.

Christensen, U., Aubert, J., 2006. Scaling properties of convection-driven dynamos in rotating spherical shells and application to planetary magnetic fields. Geophys. J. Int. 166, 97-114.

Christensen, U. R., Aubert, J., Hulot, G., 2010. Conditions for earth-like geodynamo models. Earth Planet. Sci. Lett. 296 (3), 487-496.

Christensen, U. R., Holzwarth, V., Reiners, A., 2009. Energy flux determines magnetic field strength of planets and stars. Nature 457 (7226), 167-169.

Davidson, P. A., 2013. Scaling laws for planetary dynamos. Geophys. J. Int. 195, 67-74.

Davies, C., 2015. Cooling history of earth's core with high thermal conductivity. Phys. Earth Planet. Int. $247,65-79$.

de Koker, N., Steinle-Neumann, G., Vlcek, V., 2012. Electrical resistivity and thermal conductivity of liquid Fe alloys at high P and T, and heat flux in Earth's core. Proc. Nati. Acad. Sci. USA 109 (11), 4070-4073.

Denis, C., Rybicki, K., Schreider, A., Tomecka-Suchoń, S., Varga, P., 2011. Length of the day and evolution of the earth's core in the geological past. Astronomische Nachrichten 332 (1), 24-35.

Dormy, E., Cardin, P., Jault, D., 1998. MHD flow in a slightly differentially rotating spherical shell, with conducting inner core, in a dipolar magnetic field. Earth Plan. Sci. Let. 160, 15-30.

Driscoll, P., 2016. Simulating 2 ga of geodynamo history. Geophys. Res. Lett. 43, 5680-5687. 
Driscoll, P., Bercovici, D., 2014. On the thermal and magnetic histories of earth and venus: Influences of melting, radioactivity, and conductivity. Physics of the Earth and Planetary Interiors 236, 36-51.

Driscoll, P., Evans, D., 2016. Frequency of proterozoic geomagnetic superchrons. Earth Planet. Sci. Lett. 437, $9-14$.

Evans, D., 2006. Proterozoic low orbital obliquity and axial-dipolar geomagnetic field from evaporite palaeolatitudes. Nature 444 (7115), 51-55.

Evans, D., 2013. Reconstructing pre-pangean supercontinents. Geological Society of America Bulletin 125 (1112), 1735-1751.

Gomi, H., Ohta, K., Hirose, K., Labrosse, S., Caracas, R., Verstraete, M., Hernlund, J., 2013. The high conductivity of iron and thermal evolution of the earth's core. Phys. Earth Planet. Int. 224, 88-103.

Hale, C. J., 1987. Paleomagnetic data suggest link between the Archean-Proterozoic boundary and inner-core nucleation. Nature 329 (6136), 233-237.

Heimpel, M., Evans, M., 2013. Testing the geomagnetic dipole and reversing dynamo models over earth's cooling history. Phys. Earth Planet. Int. 224, 124-131.

Hori, K., Wicht, J., Dietrich, W., 2014. Ancient dynamos of terrestrial planets more sensitive to core-mantle boundary heat flows. Planet. Space Sci. 98, 30-40.

Kent, D., Smethurst, M., 1998. Shallow bias of paleomagnetic inclinations in the paleozoic and precambrian. Earth and Planet. Sci. Lett. 160 (3), 391-402.

Konôpková, Z., McWilliams, R. S., Gómez-Pérez, N., Goncharov, A. F., 2016. Direct measurement of thermal conductivity in solid iron at planetary core conditions. Nature 534 (7605), 99-101.

Labrosse, S., 2003. Thermal and magnetic evolution of the Earth's core. Phys. Earth Planet. Int. 140, 127-143.

Labrosse, S., 2015. Thermal evolution of the core with a high thermal conductivity. Phys. Earth Planet. Int. $247,36-55$.

Landeau, M., 2013. Two aspects of fluid dynamics in planetary cores. PhD dissertation, Institut de Physique du Globe de Paris.

Landeau, M., Aubert, J., 2011. Equatorially asymmetric convection inducing a hemispherical magnetic field in rotating spheres and implications for the past martian dynamo. Phys. Earth Planet. Int. 185 (3-4), 61-73. 
Lay, T., Hernlund, J., Buffett, B. A., JAN 2008. Core-mantle boundary heat flow. Nature Geosci. 1 (1), $25-32$.

Le Bars, M., Wieczorek, M., Karatekin, Ö., Cébron, D., Laneuville, M., 2011. An impact-driven dynamo for the early moon. Nature 479 (7372), 215-218.

Lister, J. R., 2003. Expressions for the dissipation driven by convection in the Earth's core. Phys. Earth Planet. Int. 140 (1-3), 145-158.

Macouin, M., Valet, J., Besse, J., 2004. Long-term evolution of the geomagnetic dipole moment. Phys. Earth Planet. Int. 147 (2-3), 239-246.

Marti, P., Schaeffer, N., Hollerbach, R., Cébron, D., Nore, C., Luddens, F., Guermond, J.-L., Aubert, J., Takehiro, S., Sasaki, Y., et al., 2014. Full sphere hydrodynamic and dynamo benchmarks. Geophys. J. Int. 197 (1), 119-134.

McElhinny, M., 2004. Geocentric axial dipole hypothesis: a least squares perspective. Timescales of the paleomagnetic field, 1-12.

Ohta, K., Kuwayama, Y., Hirose, K., Shimizu, K., Ohishi, Y., 2016. Experimental determination of the electrical resistivity of iron at earth's core conditions. Nature 534 (7605), 95-98.

Olsen, N., Lühr, H., Finlay, C., Sabaka, T., Michaelis, I., Rauberg, J., Tøffner-Clausen, L., 2014. The chaos-4 geomagnetic field model. Geophys. J. Int. 197 (2), 815-827.

Olson, P., 1981. A simple physical model for the terrestrial dynamo. J. Geophys. Res. 86 (NB11), 875-882.

Olson, P., Aurnou, J., 1999. A polar vortex in the Earth's core. Nature 402, 170-173.

Olson, P., Christensen, U., Glatzmaier, G. A., 1999. Numerical modelling of the geodynamo: mechanisms of field generation and equilibration. J. Geophys. Res. 104 (B5), 10383-10404.

Olson, P., Christensen, U. R., 2006. Dipole moment scaling for convection-driven planetary dynamos. Earth Plan. Sci. Let. 250 (3-4), 561-571.

Olson, P., Deguen, R., Rudolph, M. L., Zhong, S., 2015. Core evolution driven by mantle global circulation. Phys. Earth Planet. Int. 243, 44-55.

O'Rourke, J., Stevenson, D., 2016. Powering earth's dynamo with magnesium precipitation from the core. Nature 529 (7586), 387-389. 
Pozzo, M., Davies, C., Gubbins, D., Alfè, D., 2013. Transport properties for liquid silicon-oxygen-iron mixtures at earth's core conditions. Phys. Rev. B 87 (1), 014110.

Roberts, P., 1988. Future of geodynamo theory. Geophysical \& Astrophysical Fluid Dynamics 44 (1-4), 3-31.

Selkin, P., Tauxe, L., 2000. Long-term variations in palaeointensity. Phil. Trans. R. Soc. Lond. A 358 (1768), $1065-1088$.

Smirnov, A., Tarduno, J., Evans, D., 2011. Evolving core conditions ca. 2 billion years ago detected by paleosecular variation. Phys. Earth Planet. Int. 187 (3), 225-231.

Smirnov, A., Tarduno, J. A., Kulakov, E., McEnroe, S., Bono, R., 2016. Paleointensity, core thermal conductivity and the unknown age of the inner core. Geophys. J. Int. 205 (2), doi: 10.1093/gji/ggw080.

Sreenivasan, B., Jones, C. A., 2005. Structure and dynamics of the polar vortex in the earth's core. Geophys. Res. Lett. 32 (20), doi: 10.1029/2005GL023841.

Stevenson, D. J., Spohn, T., Schubert, G., 1983. Magnetism and thermal evolution of the terrestrial planets. Icarus $54(3), 466-489$.

Tarduno, J., Cottrell, R., Davis, W., Nimmo, F., Bono, R., 2015. A hadean to paleoarchean geodynamo recorded by single zircon crystals. Science 349 (6247), 521-524.

Tarduno, J. A., Cottrell, R. D., Watkeys, M. K., Hofmann, A., Doubrovine, P. V., Mamajek, E. E., Liu, D., Sibeck, D. G., Neukirch, L. P., Usui, Y., 2010. Geodynamo, solar wind, and magnetopause 3.4 to 3.45 billion years ago. Science 327 (5970), 1238-1240.

Tauxe, L., 2006. Long-term trends in paleointensity: the contribution of dsdp/odp submarine basaltic glass collections. Phys. Earth Planet. Int. 156 (3), 223-241.

Valet, J. P., Besse, J., Kumar, A., Vadakke-Chanat, S., E., P., 2014. The intensity of the geomagnetic field from 2.4 ga old indian dykes. Geophys. Geochem. Geosystems. 15, 2426-2437.

Veikkolainen, T., Pesonen, L., 2014. Palaeosecular variation, field reversals and the stability of the geodynamo in the precambrian. Geophys. J. Int. 199 (3), 1515-1526.

Wang, H., Kent, D. V., Rochette, P., 2015. Weaker axially dipolar time-averaged paleomagnetic field based on multidomain-corrected paleointensities from galapagos lavas. Proc. Nat. Acad. Sci. 112 (49), 15036-15041.

Williams, G., 2000. Geological constraints on the precambrian history of earth's rotation and the moon's orbit. Rev. Geophys. 38 (1), 37-59. 


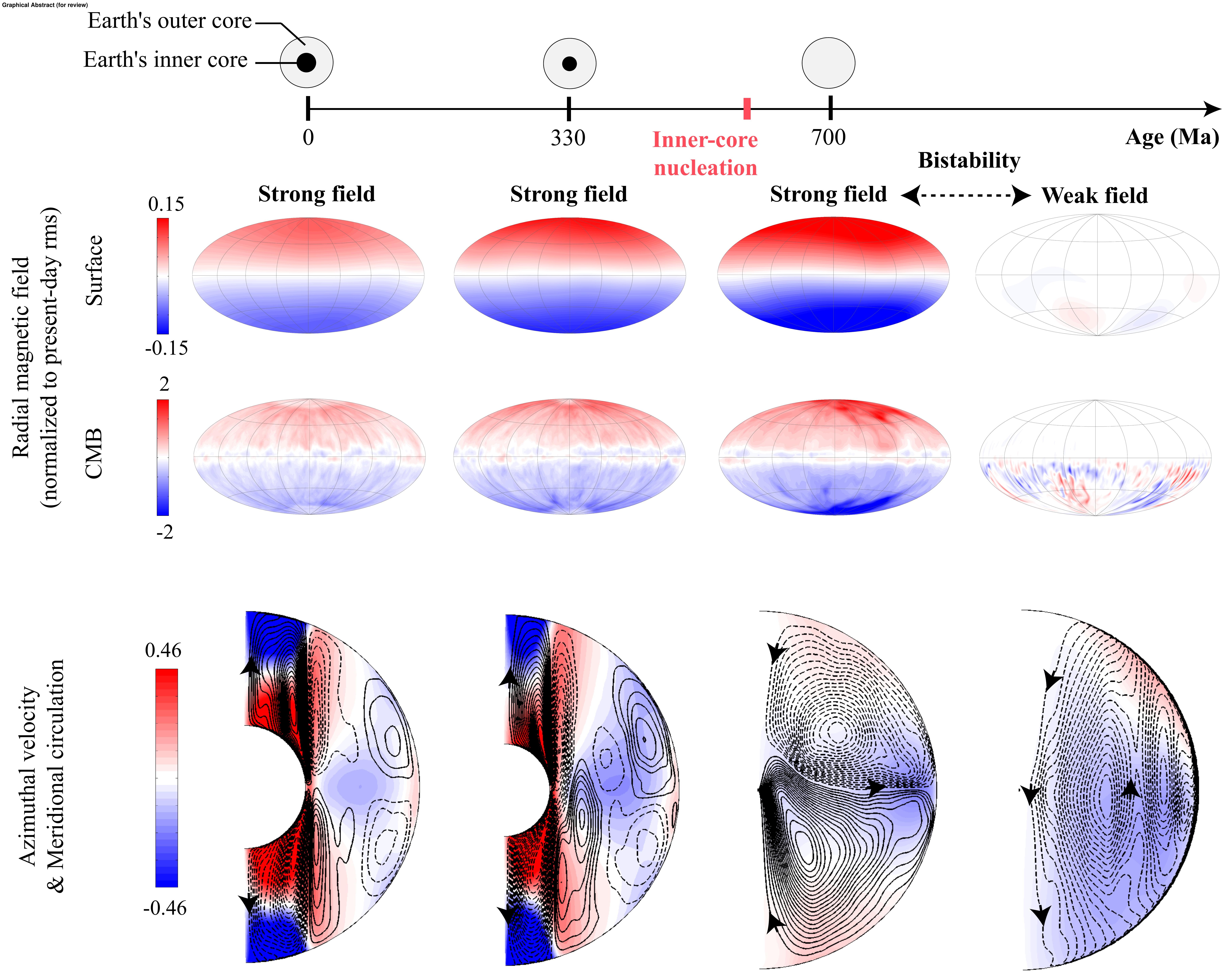



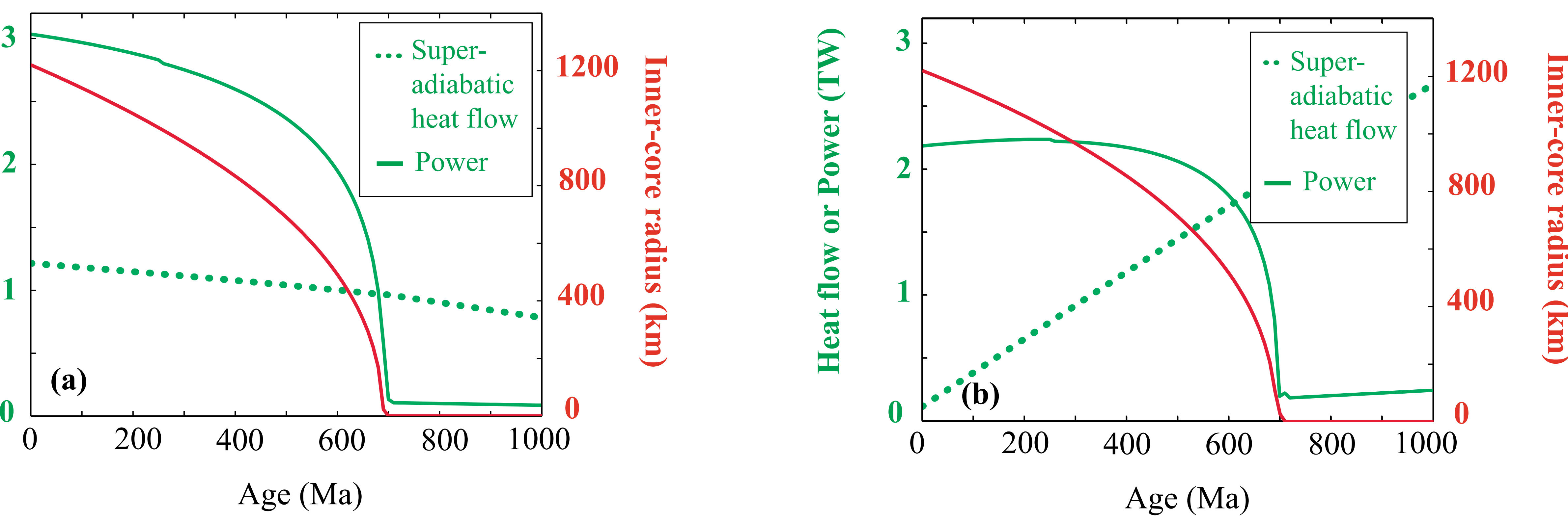

Age (Ma)

Age (Ma) 


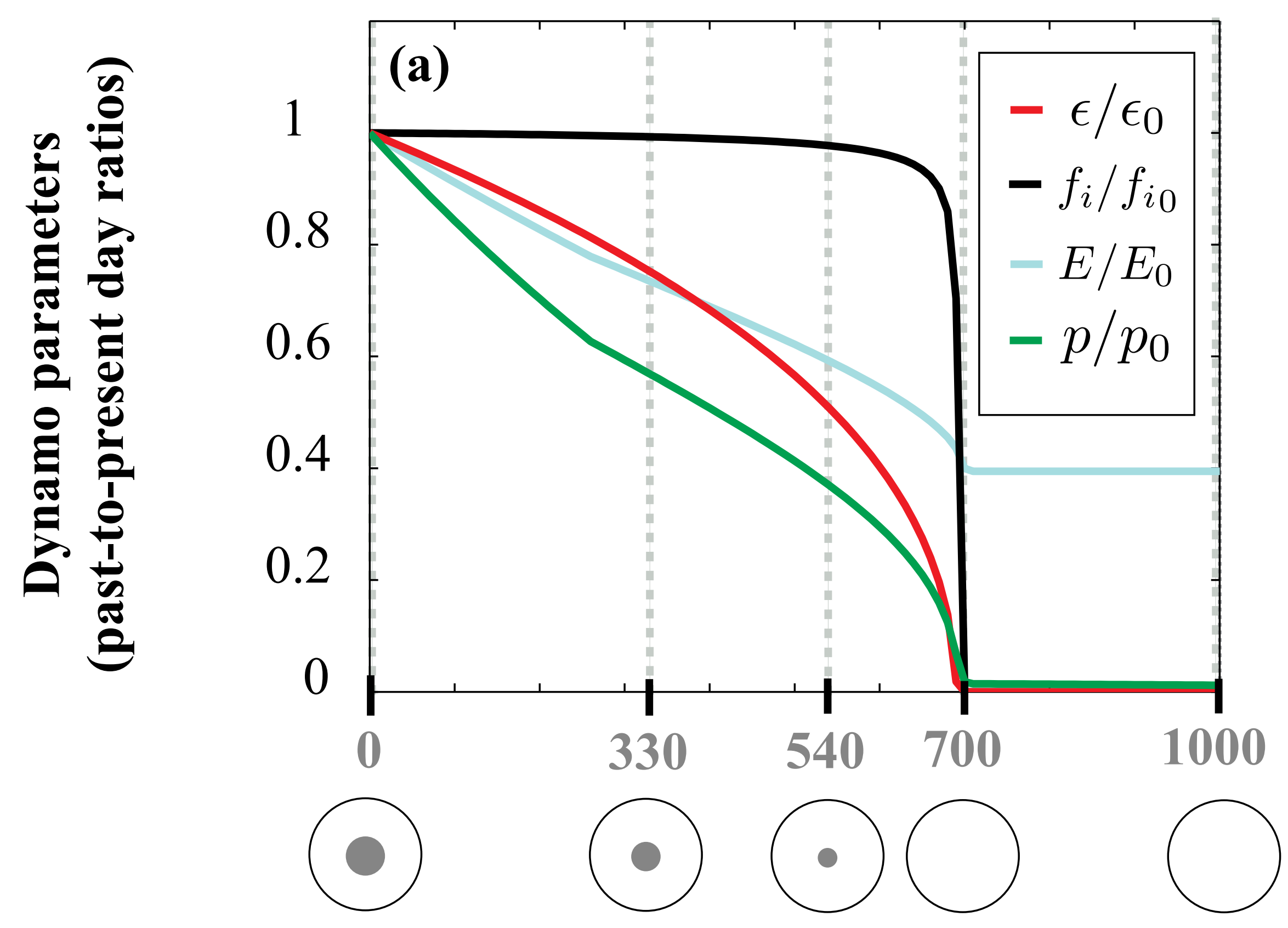

Age (Ma)

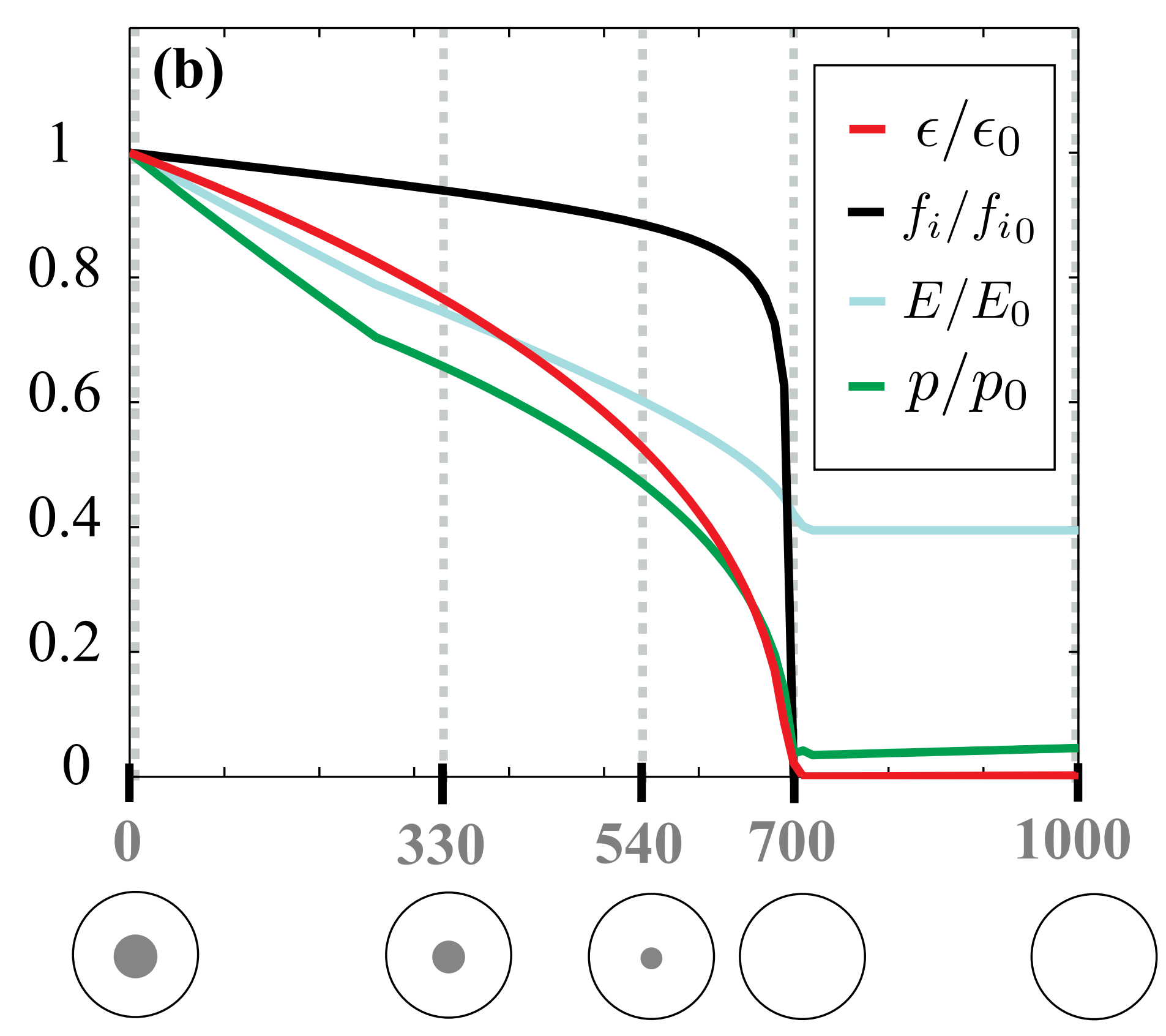

Age (Ma) 


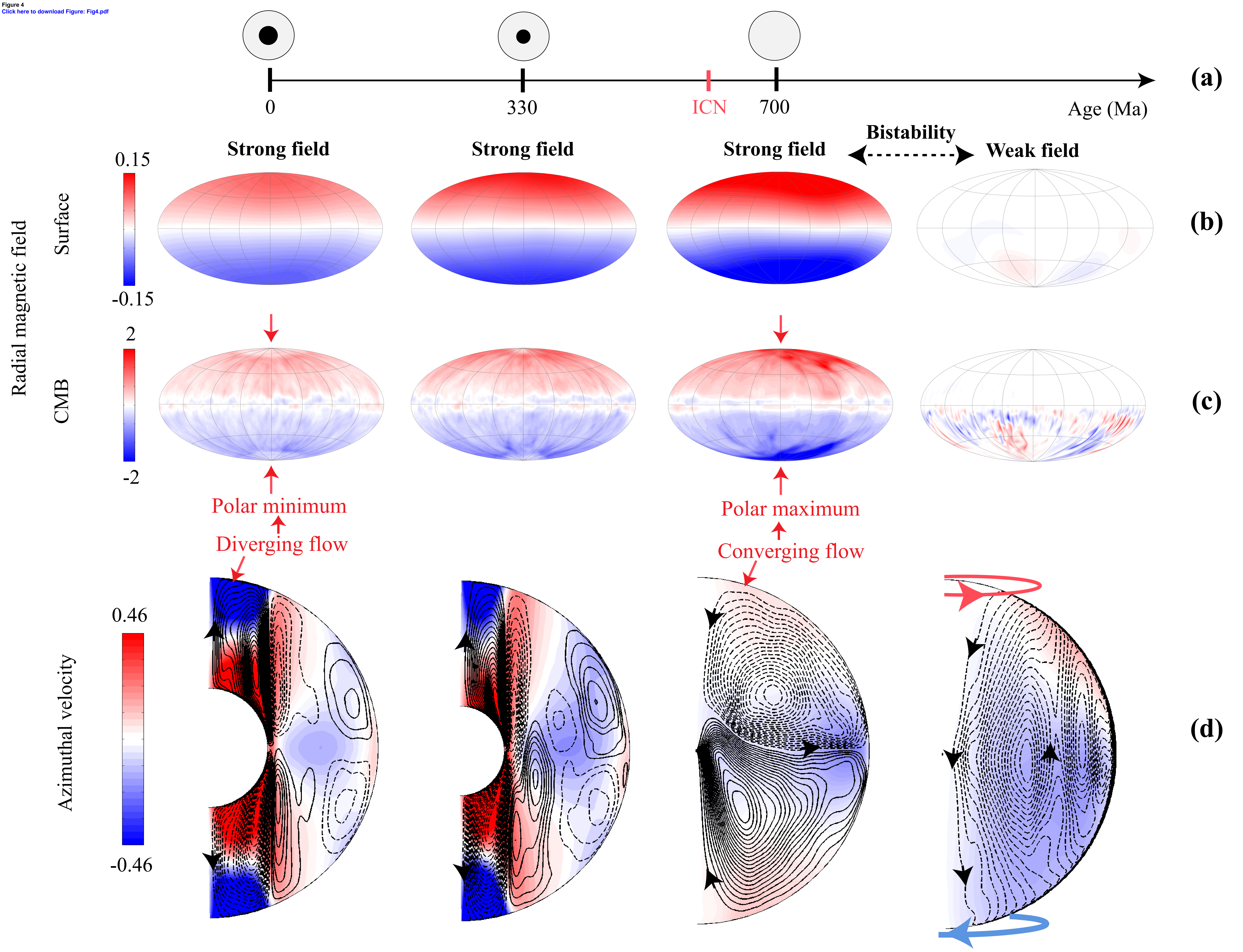




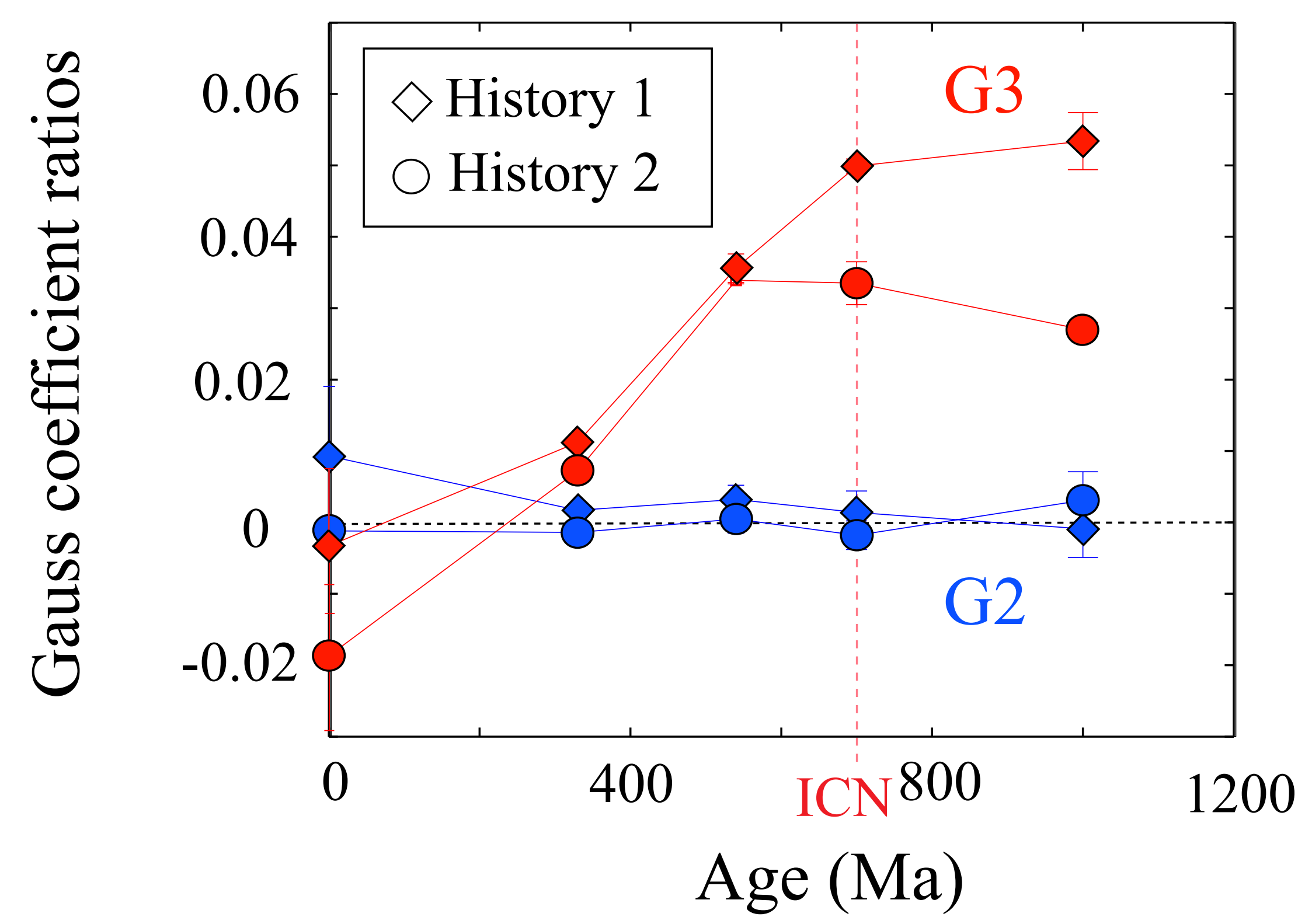

Dipole 
(b) Core History 2
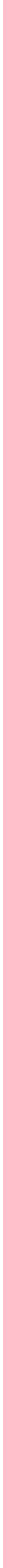
4

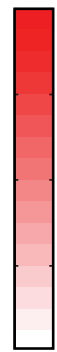

0
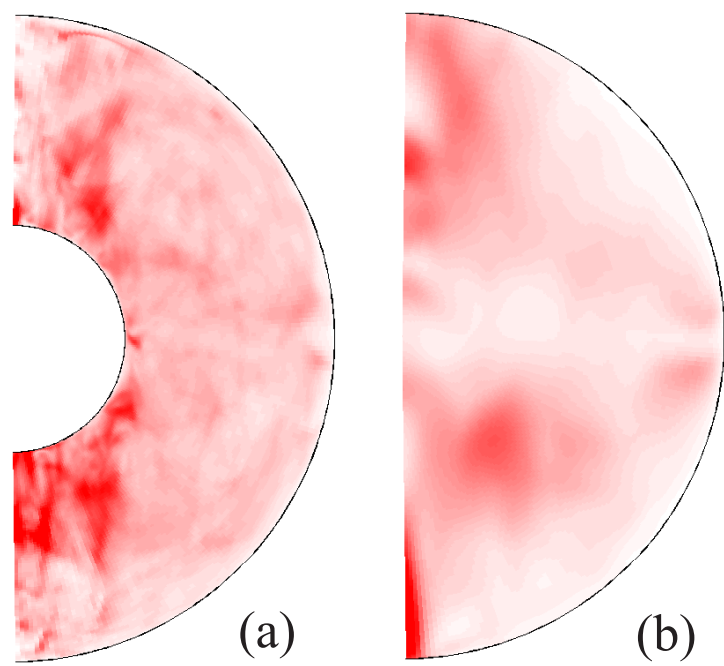

5

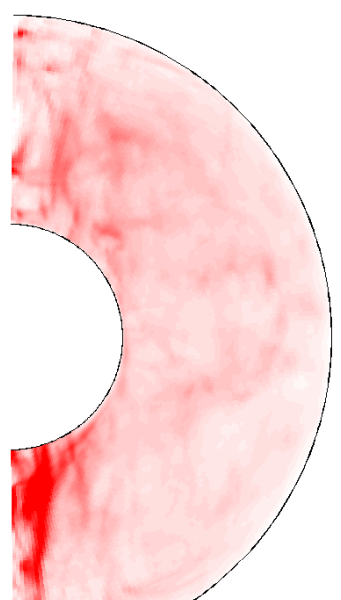

(c)

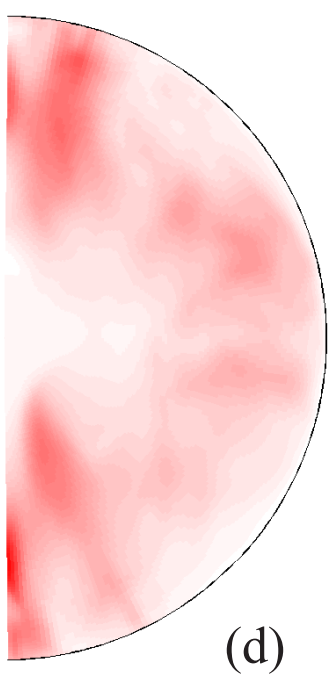

